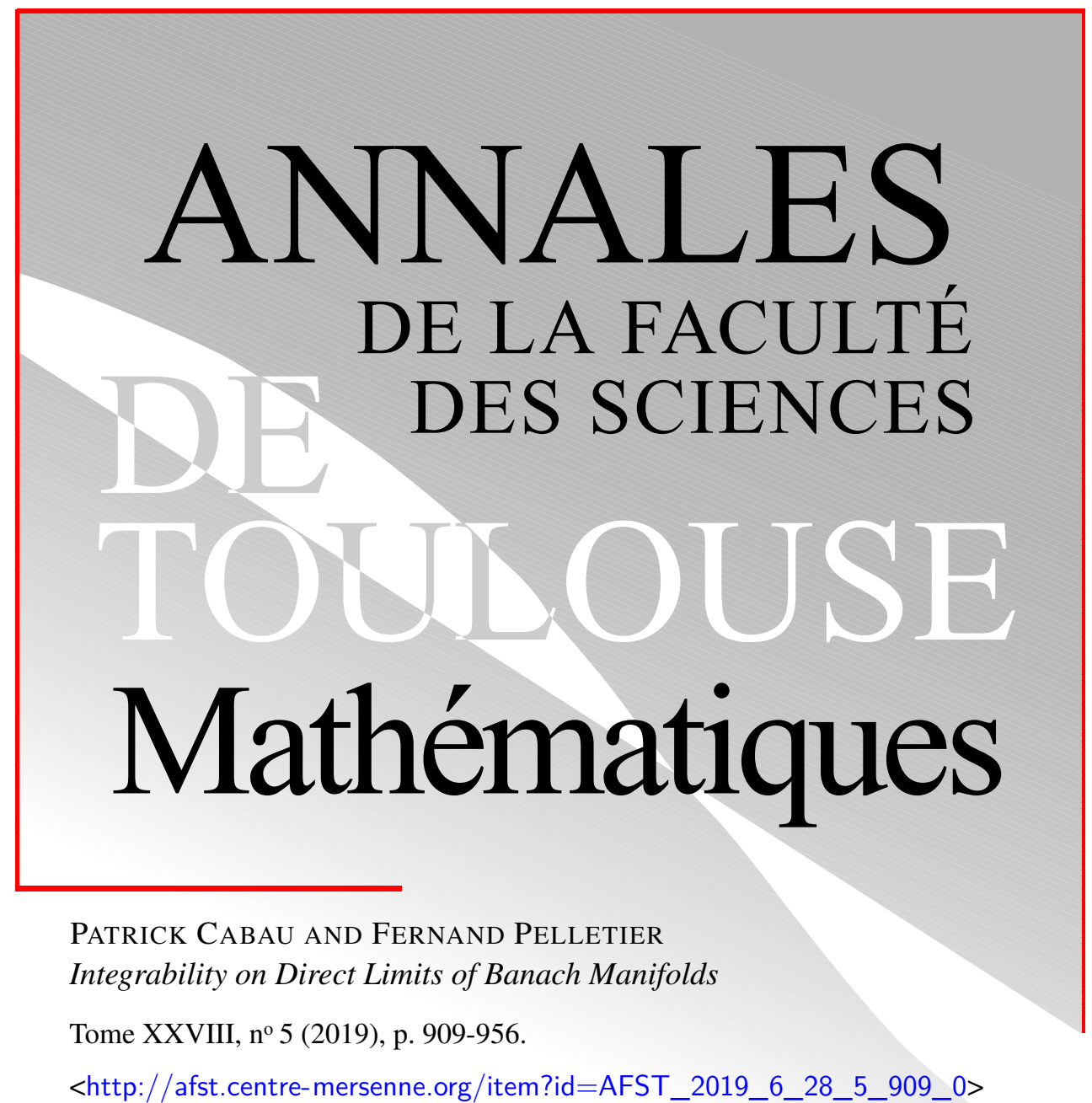

<http://afst.centre-mersenne.org/item?id=AFST_2019_6_28_5_909_0>

(C) Université Paul Sabatier, Toulouse, 2019, tous droits réservés.

L'accès aux articles de la revue «Annales de la faculté des sciences de Toulouse Mathématiques » (http://afst.centre-mersenne.org/), implique l'accord avec les conditions générales d'utilisation (http://afst. centre-mersenne.org/legal/). Toute reproduction en tout ou partie de cet article sous quelque forme que ce soit pour tout usage autre que l'utilisation à fin strictement personnelle du copiste est constitutive d'une infraction pénale. Toute copie ou impression de ce fichier doit contenir la présente mention de copyright.

\title{
cedram
}

Article mis en ligne dans le cadre du

Centre de diffusion des revues académiques de mathématiques

http://www.centre-mersenne.org/ 


\title{
Integrability on Direct Limits of Banach Manifolds ${ }^{(*)}$
}

\author{
Patrick Cabau ${ }^{(1)}$ and Fernand Pelletier ${ }^{(2)}$
}

\begin{abstract}
In this paper, we study several objects in the framework of direct limits of anchored Banach bundles over particular convenient manifolds (direct limits of Banach manifolds). In particular, we give a criterion of integrability for distributions on such convenient manifolds which are locally direct limits of particular sequences of Banach anchor ranges.

RÉsumé. - Dans cet article, on s'intéresse à l'étude de divers objets rencontrés dans le cadre de limites directes de fibrés de Banach, munis d'une ancre, au dessus de certaines variétés apparaissant comme limites directes de variétés de Banach. En particulier, on donne un critère d'intégrabilité pour des distributions sur de telles variétés qui sont localement des limites directes de suites particulières d'images d'ancres banachiques.
\end{abstract}

\section{Introduction and results}

In classical differential geometry, a distribution on a smooth manifold $M$ is an assignment

$$
\mathcal{D}: x \mapsto \mathcal{D}_{x} \subset T_{x} M
$$

on $M$, where $\mathcal{D}_{x}$ is a subspace of $T_{x} M$. This distribution is integrable if, for any $x \in M$, there exists an immersed submanifold $f: L \rightarrow M$ such that $x \in f(L)$ and for any $z \in L$, we have $T f\left(T_{z} L\right)=\mathcal{D}_{f(z)}$. On the other hand, $\mathcal{D}$ is called involutive if, for any vector fields $X$ and $Y$ on $M$ tangent to $\mathcal{D}$, their Lie bracket $[X, Y]$ is also tangent to $\mathcal{D}$.

${ }^{*}$ ) Reçu le 30 septembre 2014, accepté le 4 septembre 2017.

Keywords: Integrable distribution, direct limit, convenient structures, almost Lie Banach algebroid, almost Lie bracket, Koszul connection, anchor range.

2010 Mathematics Subject Classification: 58A30, 18A30, 46T05, 17B66, 37K30, $22 \mathrm{E} 65$.

(1) Lycée Pierre de Fermat, Parvis des Jacobins, 31000 Toulouse, France patrick.cabau@ac-toulouse.fr

(2) Lama, Université de Savoie Mont Blanc, 73376 Le Bourget du Lac Cedex, France — fernand.pelletier@univ-smb.fr

Article proposé par Jean-Pierre Otal. 
On a finite dimensional manifold, when $\mathcal{D}$ is a subbundle of $T M$, the classical Frobenius Theorem gives an equivalence between integrability and involutivity. In the other case, the distribution is singular and, even under assumptions of smoothness on $\mathcal{D}$, in general, the involutivity is not a sufficient condition for integrability (one needs some more additional local conditions). These problems were clarified and resolved essentially in [30] and [31].

In the context of Banach manifolds, the Frobenius Theorem is again true for distributions which are complemented subbundles in the tangent bundle. For singular Banach distributions closed and complemented (i.e. $\mathcal{D}_{x}$ is a complemented Banach subspace of $T_{x} M$ ) we also have the integrability property under some natural geometrical conditions (see [5] for instance). In a more general way, for weak Banach distributions $\mathcal{D}$, the integrability property is again true under some geometrical criterion (see [26] for more details).

The notion of Lie algebroid $\mathcal{A}=\left(E, \pi, M, \rho,[\cdot, \cdot]_{E}\right)$, where $\pi: E \rightarrow M$ is a fiber bundle and where the anchor $\rho$ is a morphism of Lie algebras, was first introduced by Pradines in [29]. Such objects can be seen as generalizations of both Lie algebras and tangent vector bundles. This context is an adapted framework for different problems one can meet in Mechanics (e.g. non holonomic lagrangian systems, [6]) or in symplectic Geometry in view of the symplectization of Poisson manifolds and applications to quantization $([20],[33])$.

The Stefan-Sussmann's Theorem implies the integrability of the distribution $\rho(E)$ for a finite dimensional Lie algebroid $\left(E, \pi, M, \rho,[\cdot, \cdot]_{E}\right)$. Moreover one also gets the existence of symplectic leaves for Lie-Poisson Banach manifolds under comparable assumptions. For a Banach Lie algebroid $\left(E, \pi, M, \rho,[\cdot, \cdot]_{E}\right)$ the same result is also true under some additional assumptions (see [26]).

However, the Banach context is not necessarily the most appropriate: for instance, in the framework of Lie-Poisson structure on the dual of the Lie algebra of an infinite-dimensional Lie group, the adapted model is not anymore the Banach one. A lot of infinite-dimensional Lie groups $G$, linked with symmetries depending on infinitely many parameters one can meet in Mathematical Physics, can often be expressed as the union of an ascending sequence $G_{1} \subset G_{2} \subset \cdots \subset G_{i} \subset \ldots$ of finite or infinite-dimensional Lie groups. Various examples of such objects can be found in papers of Glöckner (see [11], [12] and [14]). The convenient setting as defined by [10] and [21] seems well adapted to this framework (see for instance [11]). 
The context of this paper concerns the study of direct limits of anchored Banach bundles over direct limits of Banach manifolds endowed with convenient structures and the results of [11], [12] and [14]. More precisely, essentially we consider sequences $\left(E_{n}, \pi_{n}, M_{n}, \rho_{n}\right)_{n \in \mathbb{N}^{*}}$ of anchored Banach bundles where $\left(E_{n}, \pi_{n}, M_{n}\right)_{n \in \mathbb{N}^{*}}$ is a strong ascending sequence of Banach bundles (cf. Definition 4.17) where the anchors $\rho_{n}$ and the bonding maps $\lambda_{n}^{m}: E_{n} \rightarrow E_{m}$ and $\epsilon_{n}^{m}: M_{n} \rightarrow M_{m}$ fulfill conditions of compatibility given in Definition 6.9, (2).

Given such a sequence $\left(E_{n}, \pi_{n}, M_{n}, \rho_{n}\right)_{n \in \mathbb{N}^{*}}$, we get an anchored convenient bundle $\left(E=\underline{\lim _{1}} E_{n}, \pi=\underline{\lim _{1}} \pi_{n}, M=\underline{\lim _{1}} M_{n}, \rho=\underline{\lim _{1} \rho_{n}}\right.$ ) (cf. Theorem 6.10). Note that, according to Glöckner's results, in order to get an interesting (convenient) structure on the direct limit of Banach manifolds, an essential hypothesis is the existence of direct limit charts (cf. Definition 4.3). In particular, this assumption is true if each member $M_{n}$ of the ascending sequence $M_{1} \subset \cdots \subset M_{n} \subset \ldots$ of Banach manifolds can be endowed with a Koszul connection $\nabla^{n}$ (cf. Proposition 4.5).

Another problem in the context of direct limit of an ascending sequence $\left\{X_{n}\right\}_{n \in \mathbb{N}^{*}}$ of topological spaces is the following one: even if each $X_{n}$ is a Hausdorff topological space the direct limit $X=\underline{\lim } X_{n}$, provided with the direct limit topology, can be not Hausdorff. This leads us to introduce the notion of non necessary Hausdorff convenient manifold structure (cf. Definition 2.13).

When each $E_{n}$ can be endowed with an almost Lie bracket $[\cdot, \cdot]_{n}$ (resp. a Koszul connection $\nabla^{n}$ ) such that the restriction of $[\cdot, \cdot]_{n+1}\left(\right.$ resp. $\left.\nabla^{n+1}\right)$ to $E_{n}$ is $[\cdot, \cdot]_{n}\left(\right.$ resp. $\left.\nabla^{n}\right)$ we obtain an almost Lie bracket $[\cdot, \cdot]=\underline{\lim }[\cdot, \cdot]_{n}$ (resp. a Koszul connection $\nabla=\underline{\longrightarrow} \nabla^{n}$ ) on $E$. Moreover, if for each $n \in \mathbb{N}$ we have $\left[\rho_{n}(X), \rho_{n}(Y)\right]=\rho_{n}[X, Y]_{n}\left(\right.$ resp. $[\cdot, \cdot]_{n}$ satisfies the Jacobi identity) the same property is true for the direct limit $[\cdot, \cdot]$.

Now, according to Theorem 5 of [26], we obtain the main result of this paper:

THEOREM (Criterion of integrability (cf. Theorem 7.5)). - Let $\Delta$ be a distribution on a convenient manifold $M$ with the following properties:

(1) for any $x \in M$, there exists an open neighborhood $U$ of $x$, a strong ascending sequence of anchored Banach bundles $\left(E_{n}, \pi_{n}, U_{n}, \rho_{n}\right)_{n \in \mathbb{N}^{*}}$ endowed with a Koszul connection $\nabla^{n}$, such that $U=\underline{\lim } U_{n}$, $\underset{\mathrm{lim}}{\rho_{n}}\left(E_{n}\right)=\Delta_{\mid U}$ and such that $E_{n}$ is a complemented subbundle $\overrightarrow{o f} E_{n+1}$; 
(2) there exists an almost Lie bracket $[\cdot, \cdot]_{n}$ on $\left(E_{n}, \pi_{n}, U_{n}, \rho_{n}\right)$ such that:

- $\left(E_{n}, \pi_{n}, U_{n}, \rho_{n},[\cdot, \cdot]_{n}\right)$ is a Banach Lie algebroid;

- over each point $y_{n} \in U_{n}$ the kernel of $\rho_{n}$ is complemented in the fiber $\pi_{n}^{-1}\left(y_{n}\right)$.

Then the distribution $\Delta$ is integrable and each maximal integral manifold satisfies the direct limit chart property at any point and so is endowed with a non necessary Hausdorff convenient manifold structure.

Note that in the framework of finite-dimensional or Hilbert manifolds, this criterion of integrability requires much weaker assumptions (cf. Corollary 7.7$)$.

In order to make this article as self-contained as possible we first recall various notions: the convenient differential calculus setting as defined by Frölicher, Kriegl and Michor (Section 2), direct limits of topological vector spaces (Section 3) or manifolds (Section 4) and linear connections on Banach bundles (Section 5). In Section 6, Theorem 6.10, we prove that certain limits of Almost Lie Banach algebroids can be endowed with a structure of Almost Lie convenient algebroid. In the last part, we prove the previous theorem which is a criterion of integrability for distributions and we give an application to actions of direct limits of Banach Lie groups.

\section{Convenient differential calculus}

Differential calculus in infinite dimensions has already a long history which goes back to the beginnings of variational calculus developed by Bernoulli and Euler. During the last decades, a lot of theories of differentiation have been proposed in order to differentiate in spaces more general than Banach ones; the traditional calculus for Banach spaces is not satisfactory for the categorical point of view since the space $C^{\infty}(E, F)$ of smooth maps between Banach spaces is no longer a Banach space.

The setting of convenient differential calculus discovered by A. Frölicher and A. Kriegl (see [10]) is chosen. The reference for this section is the tome [21] which includes some further results.

In order to define the smoothness on locally convex topological vector spaces (l.c.t.v.s.) $E$, the basic idea is to test it along smooth curves (cf. Definition 2.6), since this notion in this realm is a concept without problems. 
On the one hand, a curve $c: \mathbb{R} \rightarrow E$ is differentiable if, for all $t$, the derivative $c^{\prime}(t)$ exists where $c^{\prime}(t)=\lim _{h \rightarrow 0} \frac{1}{h}(c(t+h)-c(t))$. It is smooth if all iterative derivatives exist.

On the other hand, if $J$ is an open subset of $\mathbb{R}$, we say that $c$ is Lipschitz on $J$ if the set $\left\{\frac{c\left(t_{2}\right)-c\left(t_{1}\right)}{t_{2}-t_{1}} ; t_{1}, t_{2} \in J, t_{1} \neq t_{2}\right\}$ is bounded in $E$. The curve $c$ is locally Lipschitz if every point in $\mathbb{R}$ has a neighborhood on which $c$ is Lipschitz. For $k \in \mathbb{N}$, the curve $c$ is of class $\operatorname{Lip}^{k}$ if $c$ is derivable up to order $k$, and if the $k^{t h}$-derivative $c: \mathbb{R} \rightarrow E$ is locally Lipschitz.

We then have the following link between both these notions ([21, Section 1.2]):

Proposition 2.1. - Let $E$ be a l.c.t.v.s, and let $c: \mathbb{R} \rightarrow E$ be a curve. Then $c$ is $C^{\infty}$ if and only if $c$ is Lip ${ }^{k}$ for all $k \in \mathbb{N}$.

The space $C^{\infty}(\mathbb{R}, E)$ of such curves does not depend on the locally convex topology on $E$ but only on its associated bornology (system of bounded sets). Note that the topology can vary considerably without changing the bornology; the bornologification $E_{\mathrm{born}}$ of $E$ is the finest locally convex structure having the same bounded sets.

One can note that the link between continuity and smoothness in infinite dimension is not as tight as in finite dimension: there are smooth maps which are not continuous!

The $c^{\infty}$-topology on a l.c.v.s. is the final topology with respect to all smooth curves $\mathbb{R} \rightarrow E$; it is denoted by $c^{\infty} E$. Its open sets will be called $c^{\infty}$-open.

For every absolutely convex closed bounded set $B$, the linear span $E_{B}$ of $B$ in $E$ is equipped with the Minkowski functional $p_{B}(v)=\inf \{\lambda>0: v \in \lambda . B\}$ which is a norm on $E_{B}$.

We then have the following characterization of $c^{\infty}$-open sets ([21, Theorem 2.13]):

Proposition 2.2. - $U \subset E$ is $c^{\infty}$-open if and only if $U \cap E_{B}$ is open in $E_{B}$ for all absolutely convex bounded subsets $B \subset E$.

Remark 2.3. - The $c^{\infty}$-topology is in general finer than the original topology and $E$ is not a topological vector space when equipped with the $c^{\infty}$-topology.

For Fréchet spaces and so Banach spaces, this topology coincides with the given locally convex topology. 
Definition 2.4. - A locally convex vector space $E$ is called bornological if any bounded linear mapping ${ }^{(1)} f: E \rightarrow F$ (where $F$ is any Banach space) is continuous.

LEMmA 2.5. - Let $E$ be a bornological vector space. The $c^{\infty}$-topology and the locally convex topology coincide (i.e. $c^{\infty} E=E$ ) if the closure of subsets in $E$ is formed by all limits of sequences in the subset.

Definition 2.6. - Let $E$ and $F$ be l.c.t.v.s. A mapping $f: E \rightarrow F$ is called conveniently smooth if it maps smooth curves into smooth curves, i.e. if $f \circ c \in C^{\infty}(\mathbb{R}, F)$ for all $c \in C^{\infty}(\mathbb{R}, E)$.

Note that in finite dimensional spaces $E$ and $F$ this corresponds to the usual notion of smooth mappings as proved by Boman (see [3]).

In finite-dimensional analysis, we use the Cauchy condition, as a necessary condition for the convergence of a sequence, to define completeness of the space. In the infinite-dimensional framework, we use the notion of MackeyCauchy sequence (cf. [21, Section 2]).

Definition 2.7. - A sequence $\left(x_{n}\right)$ in E is called Mackey-Cauchy if there exists a bounded absolutely convex subset $B$ of $E$ such that $\left(x_{n}\right)$ is a Cauchy sequence in the normed space $E_{B}$.

Definition 2.8. - A locally convex vector space is said to be $c^{\infty}$-complete or convenient if any Mackey-Cauchy sequence converges $\left(c^{\infty}\right.$-completeness).

We then have the following characterizations:

Proposition 2.9. - A locally convex vector space is convenient if one of the following equivalent conditions is satisfied:

(1) For every absolutely convex closed bounded set $B$ the linear span $E_{B}$ of $B$ in $E$, equipped with the norm $p_{B}$ is complete.

(2) $A$ curve $c: \mathbb{R} \rightarrow E$ is smooth if and only if $\lambda \circ c$ is smooth for all $\lambda \in E^{\prime}$ where $E^{\prime}$ is the dual consisting of all continuous linear functionals on $E$.

(3) Any Lipschitz curve in E is locally Riemann integrable.

Example 2.10. - The vector space $\mathbb{R}^{\infty}$, also denoted by $\mathbb{R}^{(\mathbb{N})}$ or $\Phi$, of all finite sequences is a countable convenient vector space $([21,47.1])$ which is not metrizable. A basis of $\mathbb{R}^{\infty}$ is $\left(e_{i}\right)_{i \in \mathbb{N}^{*}}$ where $e_{i}=\left(0, \ldots, 0,1_{i^{t h}}\right.$ term $\left.1,0, \ldots\right)$.

(1) A linear map between locally convex vector spaces is bounded if it maps every bounded set to a bounded one. 
THEOREM 2.11. - Let $U$ be a $c^{\infty}$-open set of a convenient vector space $E$ and let $F$ and $G$ be convenient vector spaces.

(1) The space $C^{\infty}(U, F)$ may be endowed with a structure of convenient vector space. The subspace $L(E, F)$ of all bounded linear mappings from $E$ to $F$ is closed in $C^{\infty}(E, F)$.

(2) The category is cartesian closed, i.e. we have the natural diffeomorphism:

$$
C^{\infty}(E \times F, G) \simeq C^{\infty}\left(E, C^{\infty}(F, G)\right) .
$$

(3) The differential operator

$$
\begin{gathered}
\mathrm{d}: C^{\infty}(E, F) \longrightarrow C^{\infty}(E, L(E, F)) \\
\mathrm{d} f(x) v=\lim _{t \rightarrow 0} \frac{f(x+t v)-f(x)}{t}
\end{gathered}
$$

exists and is linear and smooth.

(4) The chain rule holds:

$$
\mathrm{d}(f \circ g)(x) v=\mathrm{d} f(g(x)) \mathrm{d} g(x) v .
$$

Proposition 2.12. - The following constructions preserve $c^{\infty}$-completeness: limits, direct sums, strict direct limits of sequences of closed embeddings.

In general, an inductive limit of $c^{\infty}$-complete spaces needs not be $c^{\infty}$ complete (cf. [21, 2.15] for example).

According to [21, Section 27.1], a $C^{\infty}$-atlas modelled on a set $M$ modelled on a convenient space $E$ is a family $\left\{\left(U_{\alpha}, u_{\alpha}\right)\right\}_{\alpha \in A}$ of subsets $U_{\alpha}$ of $M$ and maps $u_{\alpha}$ from $U_{\alpha}$ to $E$ such that:

- $u_{\alpha}$ is a bijection of $U_{\alpha}$ onto a $c^{\infty}$-open subset of $E$ for all $\alpha \in A$;

- $M=\bigcup_{\alpha \in A} U_{\alpha}$;

- for any $\alpha$ and $\beta$ such that $U_{\alpha \beta}=U_{\alpha} \cap U_{\beta} \neq \emptyset, u_{\alpha \beta}=u_{\alpha} \circ u_{\beta}^{-1}$ : $u_{\beta}\left(U_{\alpha \beta}\right) \rightarrow u_{\alpha}\left(U_{\alpha \beta}\right)$ is a conveniently smooth map.

Classically, we have a notion of equivalent $C^{\infty}$-atlases on $M$. An equivalent class of $C^{\infty}$-atlases on $M$ is a maximal $C^{\infty}$-atlas. Such an atlas defines a topology on $M$ which is not in general Hausdorff.

Definition 2.13. - A maximal $C^{\infty}$-atlas on $M$ is called a non necessary Hausdorff convenient manifold structure on $M$ (n.n.H. convenient manifold $M$ for short); it is called a Hausdorff convenient manifold structure on $M$ when the topology defined by this atlas is a Hausdorff topological space. 
Following the classical framework, when $E$ is a Banach space (resp. a Fréchet space) we say that $M$ is a Banach manifold (resp. Fréchet manifold) if $M$ is provided with a $C^{\infty}$ - atlas (modelled on $E$ ) which generates a Hausdorff topological space.

The notion of vector bundle modelled on a convenient space over a n.n.H. convenient manifold is defined in a classic way (cf. ([21, 29]). Note that since a convenient space is Hausdorff, a vector bundle modelled on a convenient space has a natural structure of n.n.H. convenient manifold which is Hausdorff if and only if the base is a Hausdorff convenient manifold.

\section{Direct limits of topological vector spaces}

In this section the reader is referred to [4], [11] and [12].

Let $(I, \leqslant)$ be a directed set. A direct system in a category $\mathbb{A}$ is a pair $\mathcal{S}=$ $\left(X_{i}, \varepsilon_{i}^{j}\right)_{i \in I, j \in I, i \leqslant j}$ where $X_{i}$ is an object of the category and $\varepsilon_{i}^{j}: X_{i} \rightarrow X_{j}$ is a morphism (bonding map) where:

(1) $\varepsilon_{i}^{i}=\operatorname{Id}_{X_{i}}$;

(2) $\forall(i, j, k) \in I^{3}, i \leqslant j \leqslant k \Rightarrow \varepsilon_{j}^{k} \circ \varepsilon_{i}^{j}=\varepsilon_{i}^{k}$.

A cone over $\mathcal{S}$ is a pair $\left(X, \varepsilon_{i}\right)_{i \in I}$ where $X \in$ ob $\mathbb{A}$ and $\varepsilon_{i}: X_{i} \rightarrow X$ is such that $\varepsilon_{j} \circ \varepsilon_{i}^{j}=\varepsilon_{i}$ whenever $i \leqslant j$.

A cone $\left(X, \varepsilon_{i}\right)_{i \in I}$ is a direct limit of $\mathcal{S}$ if for every cone $\left(Y, \theta_{i}\right)_{i \in I}$ over $\mathcal{S}$ there exists a unique morphism $\psi: X \rightarrow Y$ such that $\psi \circ \varepsilon_{i}=\theta_{i}$. We then write $X=\underline{\lim _{\longrightarrow}} \mathcal{S}$ or $X=\underline{\lim _{\longrightarrow}} X_{i}$.

When $I=\mathbb{N}$ with the usual order relation, countable direct systems are called direct sequences.

\subsection{Direct limit of sets}

Let $\mathcal{S}=\left(X_{i}, \varepsilon_{i}^{j}\right)_{i \in I, j \in I, i \leqslant j}$ be a direct system of sets (we then have $\mathbb{A}=\mathbb{S E T})$.

Let $\mathcal{U}=\bigsqcup_{i \in I} X_{i}=\left\{(x, i): x \in X_{i}\right\}$ be the disjoint union of the sets $X_{i}$ with the canonical inclusion

$$
\begin{aligned}
\boldsymbol{\imath}_{i}: X_{i} & \longrightarrow \mathcal{U} \\
x & \longmapsto(x, i) .
\end{aligned}
$$


We define an equivalence relation on $\mathcal{U}$ as follows: $\boldsymbol{\imath}_{i}(x) \sim \boldsymbol{\imath}_{j}(y)$ if there exists $k \in I: i \leqslant k$ and $j \leqslant k$ s.t. $\varepsilon_{i}^{k}(x)=\varepsilon_{j}^{k}(y)$. We then have the quotient set $X=\mathcal{U} / \sim$ and the map $\varepsilon_{i}=\pi \circ \boldsymbol{\imath}_{i}$ where $\pi: \mathcal{U} \rightarrow \mathcal{U} / \sim$ is the canonical quotient map. Then $\left(X, \varepsilon_{i}\right)$ is the direct limit of $\mathcal{S}$ in the category $\mathbb{S E \mathbb { T }}$. If each $\varepsilon_{i}^{j}$ is injective then so is $\varepsilon_{i}$, whence $\mathcal{S}$ is equivalent to the direct system of the subsets $\varepsilon_{i}\left(X_{i}\right) \subset X$, together with the inclusion maps.

\subsection{Direct limit of topological spaces}

If $\mathcal{S}=\left(X_{i}, \varepsilon_{i}^{j}\right)_{i \in I, j \in I, i \leqslant j}$ is a direct system of topological spaces and continuous maps, then the direct limit $\left(X, \varepsilon_{i}\right)_{i \in I}$ of the sets becomes the direct limit in the category $\mathbb{T} \mathbb{O P}$ of topological spaces if $X$ is endowed with the direct limit topology ( $D L$-topology for short), i.e. the finest topology which makes the maps $\varepsilon_{i}$ continuous. So $O \subset X$ is open if and only if $\varepsilon_{i}^{-1}(O)$ is open in $X_{i}$ for each $i \in I$.

When $\mathcal{S}=\left(X_{n}, \varepsilon_{n}^{m}\right)_{n \in \mathbb{N}^{*}, m \in \mathbb{N}^{*}, n \leqslant m}$ is a direct sequence of topological spaces such that each $\varepsilon_{n}^{m}$ is injective, without loss of generality, we may assume that we have

$$
X_{1} \subset X_{2} \subset \cdots \subset X_{n} \subset X_{n+1} \subset \cdots
$$

and $\varepsilon_{n}^{n+1}$ becomes the natural inclusion. Therefore $\mathcal{S}$ will be called an ascending sequence of topological spaces and simply denoted $\left(X_{n}\right)_{n \in \mathbb{N}^{*}}$.

Moreover, if each $\varepsilon_{n}^{m}$ is a topological embedding, then we will say that $\mathcal{S}$ is a strict ascending sequence of topological spaces (expanding sequence in the terminology of [17]). In this situation, each $\varepsilon_{n}$ is a topological embedding on $X_{n}$ in $X=\lim _{n} X_{n}$.

Let us give some properties of ascending sequences of topological spaces ([12, Lemma 1.7]):

Proposition 3.1. - Let $\left(X_{n}\right)_{n \in \mathbb{N}^{*}}$ be an ascending sequence of topological spaces. Equip $X=\bigcup_{n \in \mathbb{N}^{*}} X_{n}$ with the final topology with respect to the inclusion maps $\varepsilon_{n}: X_{n} \rightarrow X$ (i.e. the DL-topology). Then we have:

(1) If each $X_{n}$ is $T_{1}$, then $X$ is $T_{1}$.

(2) If $O_{n} \subset X_{n}$ is open and $O_{1} \subset O_{2} \subset \ldots$, then $O=\bigcup_{n \in \mathbb{N}^{*}} O_{n}$ is open in $X$ and the DL-topology on $O=\lim _{\longrightarrow} O_{n}$ coincides with the topology induced by $X$.

(3) If each $X_{n}$ is locally compact, then $X$ is Hausdorff.

(4) If each $X_{n}$ is $T_{1}$ and $K \subset X$ is compact, then $K \subset X_{n}$ for some $n$. 
Unfortunately, in general, a direct limit of Hausdorff topological spaces is not Hausdorff (see [18] for an example of such a situation). Sufficient conditions on $\left(X_{n}\right)_{n \in \mathbb{N}^{*}}$ under which the direct limit $X=\bigcup_{n \in \mathbb{N}^{*}} X_{n}$ is Hausdorff can be found in [16]. However, we have:

Proposition 3.2. - Let $\left(X_{n}\right)_{n \in \mathbb{N}^{*}}$ be a strict ascending sequence of topological spaces; equip $X=\bigcup_{n \in \mathbb{N}^{*}} X_{n}$ with the DL-topology. Then we have:

(1) Assume that for each $n, X_{n}$ is closed in $X_{n+1}$.

(a) If each $X_{n}$ is normal then $X$ is normal.

(b) If each $X_{n}$ is Hausdorff and paracompact then $X$ is normal.

In particular, in each previous situation, $X$ is Hausdorff.

(2) Assume that for each $n, X_{n}$ is open in $X_{n+1}$ and Hausdorff, then $X$ is Hausdorff.

Proof.

(1a). - See [17, Proposition 4.3(i)].

(1b). - It is well known that any Hausdorff and paracompact topological space is normal and then such a topological space is Hausdorff.

(2). - See [17, Proposition 4.2].

Remark 3.3. - The direct limit of an ascending sequence $\left(X_{n}\right)_{n \in \mathbb{N}^{*}}$ is equal to the direct limit of $\left(X_{n}\right)_{n \in \mathbb{N}^{*}}, n \geqslant n_{0}$

Let $\left(X_{n}, i_{n}^{m}\right)_{n \leqslant m, m \in \mathbb{N}^{*}, n \in \mathbb{N}^{*}}$ and $\left(Y_{n}, j_{n}^{m}\right)_{n \leqslant m, m \in \mathbb{N}^{*}, n \in \mathbb{N}^{*}}$ be two ascending sequences of topological spaces. Then assume that we are given a sequence of maps $f_{n}: X_{n} \rightarrow Y_{n}$ which is consistent, i.e. that we have for any $n \leqslant m, f_{m} \circ i_{n}^{m}=j_{n}^{m} \circ f_{n}$. Then these sequences induce a map $f: X=$ $\lim _{\longrightarrow} X_{n} \rightarrow Y=\lim _{\longrightarrow} Y_{n}$ s.t. $f \circ i_{n}=i_{n} \circ f_{n}$ where $i_{n}: X_{n} \rightarrow X=\lim _{n} X_{n}$ and $j_{n}: Y_{n} \rightarrow Y=\underline{\lim } Y_{n}$ are the associate inclusions respectively.

If every map $f_{n}$ is continuous, then the induced map $f$ is continuous with respect to the $D L$-topologies on $X$ and $Y$ (continuity criterion, [19]).

\subsection{Direct limit of Banach spaces}

Let $\left(E_{n}\right)_{n \in \mathbb{N}^{*}}$ be an ascending sequence of Banach spaces. It is easy to see that we can choose a norm $\|\cdot\|_{n}$ on $E_{n}$, for $n \in \mathbb{N}^{*}$, such that:

$$
\|\cdot\|_{n+1} \leqslant\|\cdot\|_{n} \text { on } E_{n} \text { for each } n \in \mathbb{N}^{*} .
$$

In this paper, we always make such a choice. 
Given such an ascending sequence of Banach spaces, then $E=\bigcup_{n \in \mathbb{N}^{*}} E_{n}$ is called the direct limit of this sequence. The finest locally convex vector topology making each inclusion map $E_{n} \rightarrow E$ continuous, is called the locally convex direct limit topology and denoted LCDL-topology for short.

A convex set $O \subset E$ is open in this topology if and only if $O \cap E_{n}$ is open in $E_{n}$ for each $n \in \mathbb{N}^{*}$.

If $E_{n}$ is a Banach subspace of $E_{n+1}$ for each $n \in \mathbb{N}^{*}$, we have a strict ascending sequence of Banach spaces.

Definition 3.4. - A locally convex limit of ascending sequence of Banach spaces is called an (LB)-space. If the sequence is strict we speak of LB-space or strict (LB)-space.

Of course, an (LB)-space does not have a structure of convenient space in general. However, since every Banach space is convenient, then each LBspace has a structure of convenient space (see [21, Theorem 2.15]). In particular the direct limit of an ascending sequence of finite dimensional Banach spaces has a structure of convenient space.

Note that if $E=\lim _{\longrightarrow} E_{n}$ and $F=\lim _{\longrightarrow} F_{n}$ are LB-spaces, then we can identify $E \times F=\underset{\longrightarrow}{\lim }\left(E_{n} \times F_{n}\right)$ with $\underset{\lim }{\longrightarrow} E_{n} \times \underset{\lim }{ } E_{n}$ as locally convex topological spaces (cf. [19, Theorem 4.3]) and so $E \times F$ is a convenient space (cf. [21]).

We now consider a general situation which gives rise to a convenient structure on a direct limit of an ascending sequence of Banach spaces.

Proposition 3.5. - Let $\left(E_{n}\right)_{n \in \mathbb{N}^{*}}$ be an ascending sequence of Banach spaces. Assume that there exists an infinite subset $I \subset \mathbb{N}^{*}$ such that $E_{I}=$ $\bigcup_{i \in I} E_{i}=\lim _{\longrightarrow} E_{i}$ is an LB-space. Then $E=\bigcup_{n \in \mathbb{N}^{*}} E_{n}=E_{I}$ and $E$ is an LB-space.

Proof. - We set $J=\mathbb{N}^{*} \backslash I$. An index of $I$ (resp. $J$ ) will be denoted $i_{l}$, $l \in \mathbb{N}^{*}$ (resp. $j_{k}, k \in \mathbb{N}^{*}$ ). In the category of $\mathbb{S E T}$, we have $E_{I}=\bigcup_{l \in \mathbb{N}^{*}} E_{i_{l}}=$ $\lim _{\longrightarrow} E_{i_{l}}$ and $E_{J}=\bigcup_{k \in \mathbb{N}^{*}} E_{j_{k}}=\lim _{\longrightarrow} E_{j_{k}}$. From our assumption, $E_{I}$ is a con-

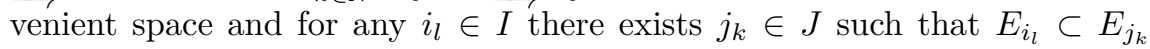
and conversely. Therefore we have the equality $E_{I}=E_{j}=E$. Thus in the category $\mathbb{S E T}$ we have

$$
E=\lim _{\longrightarrow} E_{i_{l}}=\lim _{\longrightarrow} E_{j_{k}} .
$$

Consider an open set $O=\bigcup_{l \in \mathbb{N}^{*}} O_{i_{l}}$ of $E_{i}$. Given any $j_{k} \in J$, the space $E_{j_{k}}$ is contained in some $E_{i_{l_{0}}}$ for $i_{l_{0}}<j_{k}$. Therefore $O \cap E_{j_{k}}=O \cap O_{i_{l_{0}}}$. As the inclusion $\iota_{i_{k}}^{i_{l_{0}}}: E_{j_{k}} \rightarrow E_{i_{l_{0}}}$ is continuous (as composition of a finite number 
of continuous inclusions), $O \cap E_{j_{k}}$ is an open set of $E_{j_{k}}$. It follows that the

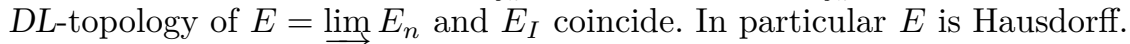

Of course, the algebraic structure of vector space on each set $E, E_{i}$ coincide and then $E$ and $E_{I}$ have the same convex sets. As a set, $O$ is an open set of the $L C D L$-topology on $E$ if and only if $O$ is convex and $O \cap E_{n}$ is open in $E_{n}$ for all $n \in \mathbb{N}^{*}$. Again, as each $E_{j_{k}}$ is contained is some $E_{i_{l_{0}}}$ for some $i_{l_{0}}>j_{k}$, it follows that $O$ is an open set of the $L C D L$-topology on $E$ if and only if $O$ is an open set for $L C D L$-topology on $E_{I}$. It follows that the $L C D L$-topology on $E$ and $E_{I}$ also coincide. Finally, the locally convex vector spaces $E_{I}$ and $E$ have the same convex bounded sets. It follows that $E$ is a convenient space with the same structure as $E_{I}$ and also the same $c^{\infty}$-topology.

LEMMA 3.6. - If $E=\lim _{\longrightarrow} E_{n}$ is an LB-space, then for the LCDLtopology we have:

(1) $E$ is Hausdorff and bounded regular (i.e. every bounded subset of E is contained in some $E_{n}$ ).

(2) Let $f: E \rightarrow F$ be a linear map where $F$ is a Banach space. The following properties are equivalent:

(a) $f$ is bounded;

(b) each restriction $f_{n}$ of $f$ to $E_{n}$ is continuous;

(c) $f$ is continuous.

Proof.

(1). - As $E$ is endowed with a convenient structure, it must be Hausdorff. On the other hand, since $E$ is an LB-space, it must be bounded regular (see for example [27]).

$(2 \mathrm{a}) \Rightarrow(2 \mathrm{~b})$. - - If $f$ is bounded, then its restriction $f_{n}$ of $f$ to $E_{n}$ is bounded, so $f_{n}: E_{n} \rightarrow F$ is continuous.

$(2 \mathrm{~b}) \Rightarrow(2 \mathrm{c})$. - Assume that each restriction $f_{n}$ of $f$ to $E_{n}$ is continuous. To prove that $f$ is continuous, it is sufficient to show that for any ball $B(0, r)$ in $F$, then $f^{-1}(B(0, r))$ is an open convex set of $E$. But we have

$$
f^{-1}(B(0, r))=\bigcup_{n \in \mathbb{N}^{*}} f_{n}^{-1}(B(0, r)) .
$$

As each $f_{n}$ is continuous, $U_{n}=f_{n}^{-1}(B(0, r))$ is an open set of $E_{n}$. Moreover, $U_{n} \subset U_{n+1}$. On the other hand, as $B(0, r)$ is convex, so is $U_{n}$. There $U=$ $f^{-1}(B(0, r))=\bigcup_{n \in \mathbb{N}^{*}} U_{n}$ is also convex. So $U$ is an open set of $E$ (relative to the $L C D L$-topology).

$(2 \mathrm{c}) \Rightarrow(2 \mathrm{a})$. - Assume now that $f$ is continuous and consider a bounded set $B$ of $E$. From part (1), $B$ is contained in some $E_{n}$. But as the inclusion 
of $E_{n}$ in $E$ is continuous, $f_{n}$ is continuous and so $f(B)=f_{n}(B)$ is bounded in $F$.

Proposition 3.7. - On an LB-space $E=\lim _{\longrightarrow} E_{n}$, the DL-topology coincides with the $c^{\infty}$-topology.

Proof. - Let $B$ be an absolutely convex bounded set of $E$. From Lemma 3.6, $B$ is contained in some $E_{n}$ and then $E_{B}$ is a vector subspace of $E_{n}$ equipped with the Minkowski norm $p_{B}$. The closed unit ball $B_{n}$ in $E_{n}$ is also an absolutely convex bounded set and there exists $\alpha \geqslant 1$ such that $B \subset \alpha \cdot B_{n}$. Remark that if $p_{n}$ is the given norm on $E_{n}$, then $p_{n}$ is the Minkowski functional associated to $B_{n}$. If $v \in E_{B}$, then $\frac{v}{p_{B}(v)}$ belongs to $\alpha . B_{n}$; so we get

$$
p_{n} \leqslant \alpha p_{B} .
$$

Therefore the inclusion of $E_{B}$ in $E_{n}$ is continuous. Let $U$ be an open set of the $D L$-topology on $E$. Then for any $n \in \mathbb{N}^{*}, U \cap E_{n}$ is open in $E_{n}$. Thus, given any absolutely convex bounded set $B$ of $E$, if $E_{B}$ is contained in $E_{n}$, then $U \cap E_{B}=U \cap E_{n} \cap E_{B}$ is open in $E_{B}$. It follows from Proposition 2.2 that $U$ is a $c^{\infty}$-open.

Conversely, if $U$ is a $c^{\infty}$-open, as $E_{n}=E_{B_{n}}$ and the norm $p_{B_{n}}$ is the given norm on $E_{n}$, again from Proposition 2.2, it follows that $U$ is an open set of the DL-topology on $E$.

Remark 3.8. - In Proposition 3.7, the fact that an LB-space is regular is essential. More generally, if an (LB)-space is convenient and bounded regular, such a result is also true. However, for the sake of simplicity, we limit ourselves to the LB-space context.

Recall that from the classical differential calculus in locally convex topological spaces, for $r \in \mathbb{N} \cup\{\infty\}$, the map $f$ is of class $C^{r}$ ( $C^{r}$-map for short) if it is continuous and, for all $k \in \mathbb{N}$ such that $k \leqslant r$, the iterated directional derivatives $\mathrm{d}_{k} f\left(x, y_{1}, \ldots, y_{k}\right):=D_{y_{1}} \ldots D_{y_{k}} f(x)$ exist for all $x \in U$ and $y_{1}, \ldots, y_{k} \in E$ and the associated $\operatorname{map}_{k} f: U \times E^{k} \rightarrow F$ is continuous. When $r=\infty$ we say that $f$ is smooth.

We have the following link between $C^{\infty}$-smoothness on each member $E_{n}$ and conveniently smoothness on $E$ for an LB-space (cf. [12, Lemma 1.9]):

Lemma 3.9. - Let $E=\lim _{E_{n}}$ be an LB-space, $U \subset E=\lim _{E_{n}}$ an open set of $E$ (for the DL-topology) and $U_{n}=U \cap E_{n}$ the associated open set in $E_{n}$. Given a map $f: U \rightarrow F$ where $F$ is a convenient space, $f$ is conveniently smooth if and only if $f_{n}=f_{\mid U \cap E_{n}}$ is $C^{\infty}$ for each $n \in \mathbb{N}^{*}$.

Proof. - This proof is an adaption of the proof of Lemma 1.9 of [12]. 
First, note that on an open set of a Banach space, we have equivalence between conveniently smoothness and $C^{\infty}$-differentiability (cf. [3]).

Assume that $f$ is conveniently smooth on $U$. Given any smooth curve $\gamma: \mathbb{R} \rightarrow U \cap E_{n}$, then $\gamma$ is a smooth curve in $U$, so $f \circ \gamma$ is smooth. As $U \cap E_{n}$ is an open set of a Banach space, it follows that $f_{n}=f_{\mid U \cap E_{n}}$ is $C^{\infty}$.

Conversely ${ }^{(2)}$ assume that $f_{n}=f_{\mid U \cap E_{n}}$ is $C^{\infty}$ and let $\gamma: \mathbb{R} \rightarrow U$ be a smooth curve. Fix $a<b$ and $k \in \mathbb{N}$. From the bounded regularity of $E$, it follows that there exists $N \in \mathbb{N}, N \geqslant n$, such that all the sets

$$
\left\{\frac{\left(\gamma^{(j)}(t)-\gamma^{(j)}(s)\right)}{s-t}:(s, t) \in\right] a, b[\times] a, b[, s \neq t\} \quad \text { and } \gamma^{(j)}(] a, b[)
$$

where $\gamma^{(j)}: \mathbb{R} \rightarrow E$ is the $j^{\text {th }}$ derivative of $\gamma$, are contained and bounded in $E_{N}$ for all $j=0, \ldots, k$. Since $\gamma_{[\mid a, b]}^{(j)}$ is a Lipschitz curve in the Banach space $E_{N}$, so there exists a primitive $\eta_{j}$ on $] a, b\left[\right.$. Thus $\eta_{j}$ and $\gamma_{\mid] a, b]}^{(j-1)}$ have the same derivative $\gamma_{\mid] a, b]}^{(j)}$ and so these curves differ by a constant for all $j=1, \ldots, k$. We conclude that $\left.\gamma^{(j-1)}:\right] a, b\left[\rightarrow E_{N}\right.$ is a $C^{1}$-curve whose derivative is $\gamma_{\mid] a, b]}^{(j)}$. As a consequence, $\gamma_{\mid] a, b]}$ is a $\operatorname{Lip}^{k}$-curve in $E_{N}$. We can choose $b-a$ small enough such that $\gamma(] a, b[) \subset U \cap E_{n} \subset U \cap E_{N}$. Since the restriction of $f$ to $U \cap E_{n}$ is smooth, it follows that, for any $\left.k \in \mathbb{N}, f \circ \gamma:\right] a, b\left[\rightarrow \mathbb{R}\right.$ is Lip $^{k}$ for any $a<b$ where $b-a$ is small enough. From Proposition 2.1, we get that $f \circ \gamma$ is smooth in $E$ and so $f$ is conveniently smooth (cf. Definition 2.6).

Proposition 3.10. - Let $E=\lim _{\underline{n}} E_{n}$ and $F=\lim _{\longrightarrow} F_{n}$ be LB-spaces and $U_{1} \subset \cdots \subset U_{n} \subset \ldots$ an ascending sequence $\left(U_{n}\right)$ of open sets of $\left(E_{n}\right)$ and set $U=\bigcup_{n \in \mathbb{N}^{*}} U_{n}$. Assume that we have a sequence of $f_{n}: U_{n}=$ $U \cap E_{n} \rightarrow F_{n}$ which are $C^{\infty}$.

(1) Then $f=\lim _{n} f_{n}$ is a conveniently smooth map from $U$ to $F$.

(2) Let $f_{n}: E_{n} \rightarrow F_{n}$ be a sequence of continuous linear maps; then $f$ is a linear map from $E$ to $F$ which is conveniently smooth and continuous for the DL-topologies.

\section{Proof.}

(1). - According to Proposition 3.1, $U$ is an open set of $E$ and $U=$ $\lim _{D} U_{n}$. So $f=\lim _{n} f_{n}: U \rightarrow F$ is a well defined continuous map (for the $D L$-topology). From Lemma $3.9, f$ is then a conveniently smooth map from $U$ to $F$.

(2) We are grateful to the anonymous referee for this part of the proof. 
(2). - Under the assumption of (2), the associated map $f=\lim _{\longrightarrow} f_{n}$ is linear and continuous. Now, as $f_{n}$ is continuous linear between Banach spaces, $f_{n}$ must be $C^{\infty}$ and by part (1), $f$ must be conveniently smooth.

\section{Direct limit of manifolds}

\subsection{Direct limit of ascending sequence of Banach manifolds}

Let $M$ be a n.n.H. convenient manifold modelled on a convenient space $E$ and $T M$ its kinematic tangent bundle (cf. [21, 28.1]). We adapt to our context the notion of weak submanifold used in [26].

Definition 4.1. - A weak submanifold of $M$ is a pair $(N, \varphi)$ where $N$ is a n.n.H. convenient connected manifold (modelled on a convenient space $F$ ) and $\varphi: N \rightarrow M$ is a conveniently smooth map such that:

- there exists a continuous injective linear map $i: F \rightarrow E$ (for the structure of l.c.v.s. of E)

- $\varphi$ is an injective conveniently smooth map and the tangent map $T_{x} \varphi: T_{x} N \rightarrow T_{\varphi(x)} M$ is an injective continuous linear map with closed range for all $x \in N$.

Note that for a weak submanifold $\varphi: N \rightarrow M$, on the subset $\varphi(N)$ of $M$, we have two topologies:

- the induced topology from $M$;

- the topology for which $\varphi$ is a homeomorphism from $N$ to $\varphi(N)$.

With this last topology, via $\varphi$, we get on $\varphi(N)$ a n.n.H. convenient manifold structure modelled on $F$. Moreover, the inclusion from $\varphi(N)$ into $M$ is continuous as a map from the manifold $\varphi(N)$ to $M$. In particular, if $U$ is an open set of $M$, then $\varphi(N) \cap U$ is an open set for the topology of the manifold on $\varphi(N)$. Therefore, if $M$ is Hausdorff so is $\varphi(N)$.

LEMMA 4.2. - Let $\mathcal{M}=\left(M_{n}\right)_{n \in \mathbb{N}^{*}}$ be an ascending sequence of Banach $C^{\infty}$-manifolds, where $M_{n}$ is modelled on the Banach space $E_{n}$ and where the inclusion $\varepsilon_{n}^{n+1}: M_{n} \rightarrow M_{n+1}$ is a $C^{\infty}$ injective map such that $\varepsilon_{n}^{n+1}\left(M_{n}\right)$ is a weak submanifold of $M_{n+1}$.

(i) There exist injective continuous linear maps $\iota_{n}^{n+1}: E_{n} \rightarrow E_{n+1}$ such that $\left(E_{n}\right)_{n \in \mathbb{N}}$ is an ascending sequence of Banach spaces.

(ii) Assume that for $x \in M=\lim _{\longrightarrow} M_{n}$, there exists a family of charts $\left(U_{n}, \phi_{n}\right)$ of $M_{n}$, for each $n \in \overrightarrow{\mathbb{N}}^{*}$, such that: 
- $\left(U_{n}\right)_{n \in \mathbb{N}^{*}}$ is an ascending sequence of chart domains;

- $\phi_{n+1} \circ \varepsilon_{n}^{n+1}=\iota_{n}^{n+1} \circ \phi_{n}$.

Then $U=\underline{\lim } U_{n}$ is an open set of $M$ endowed with the DL-topology and $\phi=\lim _{n}$ is a well defined map from $U$ to $E=\varliminf_{\longrightarrow} E_{n}$. Moreover, $\phi$ is a continuous homeomorphism from $U$ onto the open set $\phi(U)$ of $E$.

Note that, from Remark 3.3, the direct limit of $\mathcal{M}=\left(M_{n}\right)_{n \in \mathbb{N}^{*}}$ is the same as the direct limit of $\left(M_{n}\right)_{n \in \mathbb{N}^{*}, n \geqslant n_{0}}$. The result of part (ii) of this Lemma is still true if there exists an integer $n_{0}$ such that the assumptions of part (ii) are satisfied for all $n \geqslant n_{0}$.

\section{Proof.}

(i). - As $\left(M_{n}, \varepsilon_{n}^{n+1}\right)$ is a weak submanifold of $M_{n}$, there exists an injective continuous linear map $i_{n}^{n+1}: E_{n} \rightarrow E_{n+1}$ for each $n$. Therefore $\left(E_{n}\right)_{n \in \mathbb{N}^{*}}$ is an ascending sequence of Banach spaces.

(ii). - Under the assumption of part (ii), we set $V_{n}=\varphi_{n}\left(U_{n}\right)$. First, from Proposition 3.1, as $V_{n}$ is an open of $E$, we have $U=\bigcup_{n \in \mathbb{N}^{*}} U_{n}=\underline{\lim } U_{n}$ and $V=\bigcup_{n \in \mathbb{N}^{*}} V_{n}=\lim _{\longrightarrow} V_{n}$. Moreover, $U(\operatorname{resp} V)$ is an open neighborhood of $x$ (resp. $y$ ). According to the continuity criterion, $f=\underline{\lim } f_{n}$ is a continuous map from $U$ to $V$ which is injective and surjective. As each $f_{n}$ is a homeomorphism, we can apply the same arguments to the family $f_{n}^{-1}$, which ends the proof.

DeFINITION 4.3. - We say that an ascending sequence $\mathcal{M}=\left(M_{n}\right)_{n \in \mathbb{N}^{*}}$ of Banach $C^{\infty}$-manifolds has the direct limit chart property at $x \in M=$ $\lim _{\longrightarrow} M_{n}$ if $\left(M_{n}\right)_{n \in \mathbb{N}^{*}}$ satisfies the assumptions of Lemma 4.2(ii).

Once more, note that the direct limit of $\mathcal{M}=\left(M_{n}\right)_{n \in \mathbb{N}^{*}}$ is the same as the direct limit of $\left(M_{n}\right)_{n \in \mathbb{N}^{*}, n \geqslant n_{0}}$ (cf. Remark 3.3).

Example 4.4. - The existence of a direct limit chart is a natural requirement which is satisfied in many examples. We give some of them below.

(1) According to Theorem 3.1 of [12], if $\left(M_{n}\right)_{n \in \mathbb{N}^{*}}$ is an ascending sequence of $C^{\infty}$ finite dimensional manifolds, then such a sequence has the direct limit chart property at any $x \in M$.

(2) If $M$ is a compact analytic manifold, it is well known that the set $\operatorname{Diff}(M)$ of analytic diffeomorphisms of $M$ can be described as a direct limit of an ascending sequence of Banach manifolds $\left(M_{n}\right)_{n \in \mathbb{N}^{*}}$ which has the direct limit chart property for any point 
of $\operatorname{Diff}(M)$. Note that, in this case, $\left(M_{n}\right)_{n \in \mathbb{N}^{*}}$ is modelled on a sequence $\left(E_{n}\right)_{n \in \mathbb{N}^{*}}$ of Banach spaces whose direct limit $E$ is a Silva space $^{(3)}$.

(3) In [8], the reader can find examples of Lie groups which can be described as direct limits of ascending sequences of Banach manifolds $\left(M_{n}\right)_{n \in \mathbb{N}^{*}}$ modelled on sequences of Banach spaces $l^{p}$ whose direct limits $E$ are not Silva spaces.

(4) In the introduction of [14], one can also find many examples of Lie groups which have the direct limit chart property at each point.

(5) Let $\left(M_{n}\right)_{n \in \mathbb{N}^{*}}$ and $\left(N_{n}\right)_{n \in \mathbb{N}^{*}}$ be two ascending sequences of Banach manifolds which have the direct limit chart property at $x \in$ $M=\underline{\lim } M_{n}$ and at $y \in N=\underline{\lim } N_{n}$ respectively. Then $\left(M_{n} \times\right.$ $\left.N_{n}\right)_{n \in \mathbb{N}^{*}}$ has the direct limit chart property at $(x, y) \in M \times N=$ $\underline{\lim }\left(M_{n} \times N_{n}\right)$. Therefore, given any Banach manifold $M$ and any ascending sequence $\left(N_{n}\right)_{n \in \mathbb{N}^{*}}$ of $C^{\infty}$ finite dimensional manifolds, $\left(M \times N_{n}\right)_{n \in \mathbb{N}^{*}}$ has the direct limit chart property at any point of $M \times N=\underline{\longrightarrow}\left(M \times N_{n}\right)$.

We now give a general context under which an ascending sequence $\mathcal{M}=$ $\left(M_{n}\right)_{n \in \mathbb{N}^{*}}$ of Banach $C^{\infty}$-manifolds has the direct limit chart property at each point of $M=\underline{\lim } M_{n}$.

Proposition 4.5. - Let $\mathcal{M}=\left(M_{n}\right)_{n \in \mathbb{N}^{*}}$ be an ascending sequence of Banach $C^{\infty}$-manifolds modelled on the ascending sequence $\left(E_{n}\right)_{n \in \mathbb{N}^{*}}$. We assume that:

(i) $E_{n}$ is a complemented subspace in $E_{n+1}$ for each $n \in \mathbb{N}^{*}$;

(ii) there exists a linear connection ${ }^{(4)}$ on $T M_{n}$ for each $n \in \mathbb{N}^{*}$.

Then $E=\lim _{\longrightarrow} E_{n}$ is an LB-space and $\mathcal{M}$ has the direct limit chart property at each point of $M=\underline{\lim } M_{n}$.

Example 4.6. - By application of Proposition 4.5, $\mathcal{M}=\left(M_{n}\right)_{n \in \mathbb{N}^{*}}$ has the direct limit chart property at each point in the following cases:

(1) Each space $M_{n}$ is a paracompact finite dimensional manifold (cf. [12]).

(2) Each manifold $M_{n}$ is a smooth paracompact ${ }^{(5)}$ Hilbert submanifold of $M_{n+1}$.

(3) A locally convex limit of ascending sequence of Banach spaces where each inclusion is compact is called a Silva space or a (DFS)-space (for dual of Fréchet Schwartz space).

(4) For the definition of a connection on a Banach manifold see for instance [9]. For more details see also Subsection 5.2.

${ }^{(5)}$ i.e. $M_{n}$ is paracompact and every locally finite open covering of $M_{n}$ admits a conveniently smooth partition of unity subordinated to it. 
(3) Each manifold $M_{n}$ is a smooth paracompact Banach submanifold of $M_{n+1}$.

The proof of Proposition 4.5 requires the following Lemma:

Lemma 4.7. - Let $N_{1}$ be a Banach complemented immersed submanifold, modelled on $F_{1}$, of a Banach manifold $N$, modelled on $F$ with $F_{1} \subset F$. Assume that there exists a linear connection on $T N$. Given any chart $\left(U_{1}, \phi_{1}\right)$ of $x$ in $N_{1}$ such that $U_{1}$ is a contractible set, there exists a chart $(U, \Phi)$ of $x \in N_{1}$ such that $U$ is contractible, $U \cap N_{1}=U_{1}$ and $\Phi_{\mid U_{1}}=\phi_{1}$.

Proof. - Let $N_{1}$ be an immersed complemented submanifold of a Banach manifold $N$. If $N_{1}$ (resp. $N$ ) is modelled on $F_{1}$ (resp. $F$ ), there exists a Banach subspace $F_{2}$ of $F$ such that $F=F_{1} \oplus F_{2}$.

Assume that there exists a linear connection on $T N$. Therefore we have an exponential map Exp : $\mathcal{O} \subset T N \rightarrow N$ where $\mathcal{O}$ is an open neighborhood of the zero section in $T N$. Note that $\operatorname{Exp}_{\mid T_{x} N}$ is a local diffeomorphism.

Choose a chart $\left(U_{1}, \phi_{1}\right)$ of $N_{1}$ around $x$ such that $U_{1}$ is a contractible set. Now, as $N_{1}$ is immersed in $N$, for each $z \in U_{1}$, there exists a chart $\left(U_{z}, \Phi_{z}\right)$ of $N$ such that

$$
\Phi_{z}\left(U_{z} \cap N_{1}\right)=\phi_{1}\left(U_{z} \cap U_{1}\right) \times\left\{0_{F_{2}}\right\} \quad \text { and } \quad \Phi_{\left.z\right|_{U_{z} \cap U_{1}}}=\phi_{1 \mid U_{z} \cap U_{1}}
$$

Then $U=\bigcup_{z \in U_{1}} U_{z}$ is an open neighborhood containing $U_{1}$ and we have

$$
U \cap N_{1}=\bigcup_{z \in U_{1}}\left(U_{z} \cap N_{1}\right)=U_{1} .
$$

Therefore $U_{1}$ is a closed submanifold of $U$.

As $U_{1}$ is a contractible set, the restriction of $T N$ to $U_{1}$ is trivial (cf. [1, Theorem 3.4.35]). Therefore we have a diffeomorphism $\Theta: T N_{\mid U_{1}} \rightarrow U_{1} \times F$. In the trivial bundle $U_{1} \times F$, we can consider the subbundle $U_{1} \times F_{2}$ and we have $T N_{\mid U_{1}}=T U_{1} \oplus \Theta^{-1}\left(U_{1} \times F_{2}\right)$. As $\phi_{1}(U)$ is an open set of $F_{1}$ and $F_{1}$ is paracompact, so is $U_{1}$. Therefore by same arguments used in the proof of Theorem 5.1 of [22, Chapter IV], we can build a diffeomorphism $\Psi$ from an open neighborhood $\mathcal{U}$ of the zero section of $\Theta^{-1}\left(U_{1} \times F_{2}\right)$ on an open neighborhood $U$ of $U_{1}$ in $N$. Note that $\mathcal{U}$ is a fibration on the zero section of $\Phi^{-1}\left(U_{1} \times F_{2}\right)$. Moreover, from the property of Exp, we can choose $\mathcal{U}$ such that each fiber is a contractible set. We denote by $\Phi$ the composition defined by $\Phi^{-1}=\Theta \circ \Psi^{-1} \circ\left(\left(\phi_{1}\right)^{-1} \times \operatorname{Id}_{F_{2}}\right)$. As $\operatorname{Exp}_{\mid T_{u} N}(0)(u)=u$ we finally have $\Phi^{-1}(v, 0)=\left(\phi_{1}\right)^{-1}(v)$.

Proof of Proposition 4.5. - We have already seen that $E=\underline{\lim _{\longrightarrow}} E_{n}$ is an LB-space (cf. Proposition 2.12). 
Now consider any point $x \in M=\underline{\lim } M_{n}$. Then $x$ belongs to some $E_{n}$. Let $l_{0}$ be the first integer $l$ such that $x$ belongs to $M_{l}$. Assume that for each integer $l_{0} \leqslant l \leqslant k$ we have the following property: there exists a family of charts $\left(U_{n}, \phi_{n}\right)$ of $M_{n}$, for each $l_{0} \leqslant n \leqslant l$, such that:

- $\left(U_{n}\right)_{l_{0} \leqslant n \leqslant l}$ is an ascending sequence of chart domains around $x$;

- $\phi_{n+1 \mid U_{n}}=\phi_{n}$ for all $l_{0} \leqslant n<l$.

From Lemma 4.7, this assumption is true for $l=l_{0}+1$. The proof is obtained by induction using Lemma 4.7 .

Now we can prove the following result which generalizes [12, Theorem 3.1].

THEOREM 4.8. - Let $\left(M_{n}\right)_{n \in \mathbb{N}^{*}}$ be an ascending sequence of Banach $C^{\infty}$-manifolds, modelled on the Banach spaces $E_{n}$. Assume that $\left(M_{n}\right)_{n \in \mathbb{N}^{*}}$ has the direct limit chart property at each point $x \in M=\underline{\lim } M_{n}$ and $E=\lim _{\longrightarrow} E_{n}$ is an LB-space. Then there is a unique n.n.H. convenient manifold structure on $M=\lim M_{n}$ modelled on the convenient space $E$ such that the topology associated to this structure is the DL-topology on $M$. In particular, for each $n \in \mathbb{N}^{*}$, the canonical injection $\varepsilon_{n}: M_{n} \rightarrow M$ is an injective conveniently smooth map and $\left(M_{n}, \varepsilon_{n}\right)$ is a weak submanifold of $M$. Moreover, if each $M_{n}$ is locally compact or is open in $M_{n+1}$ or is a paracompact Banach manifold closed in $M_{n+1}$, then $M=\underline{\lim } M_{n}$ is provided with a Hausdorff convenient manifold structure.

A direct application of this theorem gives rise to the following result:

Corollary 4.9. - Let $\left(M_{n}\right)_{n \in \mathbb{N}^{*}}$ be an ascending sequence of Banach paracompact $C^{\infty}$-manifolds where $M_{n}$ is closed in $M_{n+1}$. If the sequence $\left(M_{n}\right)_{n \in \mathbb{N}^{*}}$ satisfies the assumptions of Proposition 4.5, then $M=\lim _{\longrightarrow} M_{n}$, provided with the DL- topology, has a unique structure of Hausdorff convenient manifold modelled on an LB-space.

Proof of Theorem 4.8. - As in Lemma 4.2(ii), we consider the set $\mathcal{A}$ of all sequences of charts $\left\{\left(U_{n}^{\alpha}, \phi_{n}^{\alpha}\right)_{n \in \mathbb{N}^{*}}\right\}_{\alpha \in A}$ of $\left(M_{n}\right)_{n \in \mathbb{N}^{*}}$ such that $\left(U_{n}\right)$ is an ascending sequence of chart domains. We set $V_{n}^{\alpha}=\phi_{n}^{\alpha}\left(U_{n}^{\alpha}\right), U^{\alpha}=\underline{\lim } U_{n}^{\alpha}$ and $\phi^{\alpha}=\lim _{n} \phi_{n}^{\alpha}$. From Lemma $4.2(\mathrm{ii}), \phi^{\alpha}$ is a homeomorphism from ${\overrightarrow{U^{\alpha}}}$ to the open set $V^{\alpha}=\phi^{\alpha}\left(U^{\alpha}\right)=\lim _{n} V_{n}^{\alpha}$ of $E$. Then $U^{\alpha}$ and $V^{\alpha}$ are open sets of the $D L$-topology on $M$ and $E$ respectively (cf. Proposition 3.1(2)). From our assumption, $\mathcal{A}$ is then a topological atlas of $M$ modelled on the convenient space $E$. Note that, from Proposition 3.7, each $V^{\alpha}$ is also a $c^{\infty}$-open set.

Let us prove that the change of charts are conveniently smooth diffeomorphisms. Consider two charts $\left(U^{\alpha}, \phi^{\alpha}\right)$ and $\left(U^{\beta}, \phi^{\beta}\right)$ around $x \in M$. We 
consider

$$
\tau_{n}^{\beta \alpha}=\phi_{n}^{\beta} \circ\left(\phi_{n}^{\alpha}\right)^{-1}: \phi_{n}^{\alpha}\left(U_{n}^{\alpha} \cap U_{n}^{\beta}\right) \longrightarrow \phi_{n}^{\beta}\left(U_{n}^{\alpha} \cap U_{n}^{\beta}\right) .
$$

For each $n \in \mathbb{N}^{*}$, the pairs $\left(U_{n}^{\alpha}, \phi_{n}^{\alpha}\right)$ and $\left(U_{n}^{\beta}, \phi_{n}^{\beta}\right)$ are charts of $M_{n}$ and the intersection of their domains is not empty. It follows that the map $\tau_{n}^{\beta \alpha}$ is a $C^{\infty}$ local diffeomorphism of $E_{n}$. But the construction of $U^{\alpha}$ and $U^{\beta}$ implies that $U^{\alpha} \cap U^{\beta}$ is the direct limit of $\left(U_{n}^{a} \cap U_{n}^{\beta}\right)_{n \in \mathbb{N}^{*}}$ in $M$. It follows that $\phi^{\alpha}\left(U^{\alpha} \cap U^{\beta}\right)$ is the direct limit of $\phi_{n}^{\alpha}\left(U_{n}^{\alpha} \cap U_{n}^{\beta}\right)_{n \in \mathbb{N}^{*}}$. In the same way, we have $\phi^{\alpha}\left(U^{\alpha} \cap U^{\beta}\right)=\lim _{n} \phi_{n}^{\alpha}\left(U_{n}^{\alpha} \cap U_{n}^{\beta}\right)$. Therefore we get a direct limit map $\tau^{\beta \alpha}=\lim _{n} \tau_{n}^{\beta \alpha}$ from the open set $\phi^{\alpha}\left(U^{\alpha} \cap U^{\beta}\right)$ onto the open set $\phi^{\beta}\left(U^{\alpha} \cap U^{\beta}\right)$ of $E$. Again the sets $\phi^{\alpha}\left(U^{\alpha} \cap U^{\beta}\right)$ and $\phi^{\beta}\left(U^{\alpha} \cap U^{\beta}\right)$ are $c^{\infty}$-open sets of $E$. As each $\tau_{n}^{\beta \alpha}$ is a $C^{\infty}$ diffeomorphism of $E_{n}$, Lemma 3.9 implies that $\tau^{\alpha \beta}$ is a conveniently smooth diffeomorphism from $\phi^{\alpha}\left(U^{\alpha} \cap U^{\beta}\right)$ onto $\phi^{\beta}\left(U^{\alpha} \cap U^{\beta}\right)$. Therefore we obtain that $\mathcal{A}$ is convenient atlas on $M$. Note that the topology of $M$ defined by such an atlas is exactly the $D L$-topology on $M$. Therefore, if $M_{n}$ locally compact, then $M$ is Hausdorff from Proposition 3.1. In the same way, from Proposition 3.2, if $M_{n}$ is open in $M_{n+1}$ or is paracompact and closed in $M_{n+1}$ for each $n$, then $M$ is Hausdorff. Thus in each of the previous particular cases, $M$ is provided with a Hausdorff convenient manifold structure.

Now we prove the uniqueness of this convenient structure. Assume that $Y$ is a convenient manifold structure modelled on the convenient vector space $E$ and $h_{n}: M_{n} \rightarrow Y$ a $C^{\infty}$ map for each $n \in \mathbb{N}^{*}$ s.t. $\left(Y,\left(h_{n}\right)_{n \in \mathbb{N}^{*}}\right)$ is a cone over $\mathcal{S}$. Then there is a uniquely determined continuous map $h: M \rightarrow Y$ s.t. $h_{\mid M_{n}}=h_{n}$. Let $x \in M$; we can find a chart $(U, \varphi)$ around $x$ in the atlas $\mathcal{A}$ where $f=\lim _{n} f_{n} U$ for charts $\varphi_{n}: U_{n} \rightarrow E_{n}$. Let $\psi: W \subset Y \rightarrow V$ be a chart for $Y(W$ is an open set of $Y)$. Then $O=\left(h \circ \varphi^{-1}\right)^{-1}(W)$ is an open set of $U \subset E$ and $O_{n}=O \cap E_{n}$ is open in $E_{n}$ for each $n$. Consider $g=\left.\psi \circ h \circ \varphi^{-1}\right|_{O} ^{V}: O \rightarrow V$. Then $g_{\mid O_{n}}=\left.\psi \circ h_{n} \circ \varphi_{n}^{-1}\right|_{O_{n}} ^{V}: O_{n} \rightarrow V$ is $C^{\infty}$ for each $n \in \mathbb{N}^{*}$. Hence $g$ is $c^{\infty}$ (cf. Proposition 3.10), so is $h$ on the open neighborhood $U$ of $x$ and hence on all of $M$ because $x$ is arbitrary. Thus $\left(M,\left(\varepsilon_{n}\right)_{n \in N^{*}}\right)=\lim _{\longrightarrow} S$ in the category of $c^{\infty}$-manifolds. The uniqueness of a convenient structure of manifold on $M$ follows from the universal property of direct limits.

\subsection{Direct limit of Lie groups}

The reader is referred to [14].

Interesting infinite-dimensional Lie groups often appears as direct limits $G=\bigcup_{n \in \mathbb{N}^{*}} G_{n}$ of ascending Lie groups $G_{1} \subset G_{2} \subset \ldots$ where the bonding 
maps (inclusion maps $\varepsilon_{n}^{n+1}: G_{n} \rightarrow G_{n+1}$ ) are smooth homomorphisms (e.g. the group $\operatorname{Diff}_{c}(M)$ of compactly supported diffeomorphisms of a $\sigma$-compact smooth manifold $M$ or the test function groups $C_{c}^{\infty}(M, H)$ of compactly supported smooth maps with values in a finite-dimensional Lie group $H$ ).

When the Lie groups $G_{n}$ are finite dimensional it is well known that the direct limit $\lim _{\longrightarrow} G_{n}$ can be endowed with a structure of Lie group (see [12]).

Here we give conditions on the direct sequences $\mathcal{G}=\left(G_{n}, \varepsilon_{n}^{m}\right)_{n, m \in \mathbb{N}^{*}, n \leqslant m}$ of Lie groups in order to obtain a structure of Lie group on their direct limit.

We first recall the essential notion of candidate for a direct limit chart:

DEFINITION 4.10. - Let $G=\bigcup_{n \in \mathbb{N}^{*}} G_{n}$ be the union of an ascending sequence of $C^{\infty}$-Lie groups $G_{n}$ where the inclusion maps $\varepsilon_{n}^{m}: G_{n} \rightarrow G_{m}$ are $C^{\infty}$-homomorphisms and $G_{n}$ is a subgroup of $G$.

We say that $G$ has a candidate for a direct limit chart if there exist charts $\phi_{n}: G_{n} \supset U_{n} \rightarrow V_{n} \subset \mathfrak{g}_{n}$ of $G_{n}$ around the identity for $n \in \mathbb{N}^{*}$ (where $\mathfrak{g}_{n}$ stands for the Lie algebra of $\left.G_{n}\right)$ such that $U_{n} \subset U_{m}$ and $\left.\phi_{m}\right|_{U n}=\mathbb{L}\left(i_{n}^{m}\right) \circ \phi_{n}$ if $n \leqslant m$ and $V=\bigcup_{n \in \mathbb{N}^{*}} V_{n}$ is open in the locally convex direct limit $\lim _{\mathfrak{g}_{n}}$ which we assume to be Hausdorff.

Glöckner obtains the following result (cf. [14, Proposition 1.4.3]):

Proposition 4.11. - Let $G=\bigcup_{n \in \mathbb{N}^{*}} G_{n}$ be a group which is the union of an ascending sequence of $C^{\infty}$-Lie groups. Assume that $G$ has a candidate $\phi: U \rightarrow V \subset \lim _{\mathfrak{n}} \mathfrak{g}_{n}$ for a direct limit chart and assume that one of the following conditions is satisfied:

(i) $G_{n}$ is a Banach Lie group for each $n \in \mathbb{N}^{*}$ and the inclusion map $\mathfrak{g}_{n} \rightarrow \mathfrak{g}_{m}$ is a compact linear operator for all $n<m$;

(ii) $\mathfrak{g}_{n}$ is a $k_{\omega}-$ space for each $n \in \mathbb{N}^{*}$.

Then on $G=\lim _{\longrightarrow} G_{n}$ there exists a unique $C^{\infty}$-Lie group structure making $\phi_{\mid W}$ a direct chart limit for $G$ around 1 , where $W$ is an open neighbourhood of 1 contained in $U$.

Glöckner gives also results in the convenient setting where $V=\bigcup_{n \in \mathbb{N}^{*}} V_{n}$ is a $c^{\infty}$-open set in $\lim _{\longrightarrow} \mathfrak{g}_{n}$ endowed with a suitable locally convex topology (cf. [13, Remark 14.8]):

Proposition 4.12. - Let $G=\bigcup_{n \in \mathbb{N}^{*}} G_{n}$ be a group which is the union of an ascending sequence of (Hausdorff) convenient Lie groups. Equip the vector space $\lim _{\mathfrak{n}} \mathfrak{g}_{n}$ with the locally convex vector topology associated with the direct limit bornology which is assumed to be Hausdorff. We require that 
$G$ admits a candidate for a direct limit chart in the convenient sense and that each bounded subset in $\mathfrak{g}$ is a bounded subset of some $\mathfrak{g}_{n}$. Then $G$ may be endowed with a structure n.n.H. convenient Lie group

This criterion permits to obtain:

THEOREM 4.13. - Let $G=\bigcup_{n \in \mathbb{N}^{*}} G_{n}$ be a group which is the union of an ascending sequence of Banach Lie groups. Assume that the direct limit $\mathfrak{g}=\underline{\lim } \mathfrak{g}_{n}$ of the ascending sequence $\left(\mathfrak{g}_{n}\right)_{n \in \mathbb{N}^{*}}$ of associated Lie algebras is

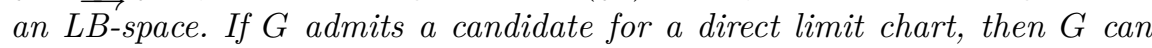
be endowed with a structure of n.n.H. convenient Lie group modelled on the LB-space $\mathfrak{g}$.

Therefore, Proposition 4.11(ii) can be seen as a corollary of this theorem. According to Proposition 3.5, if $G$ admits a candidate for a direct limit chart then assume that there exists a countable subset $I \subset \mathbb{N}^{*}$ such that the direct limit $\mathfrak{g}_{I}=\lim _{\mathfrak{l}}\left\{\mathfrak{g}_{i}, i \in I\right\}$ is an LB-space, then $G$ is endowed with a structure of convenient Lie group modelled on the LB-space $\mathfrak{g}$. Now since $\mathfrak{g}_{n}$ is a $k_{\omega}$-space for each $n \in \mathbb{N}^{*}$, by direct chart limit property and using the fact that direct limits of ascending sequences of locally $k_{\omega}$-spaces are locally $k_{\omega^{-}}$ spaces and so are Hausdorff (see [14]), the topology on $G$ must be Hausdorff.

On the other hand, the reader can find the following criterion in [8]:

THEOREM 4.14. - Let $\mathcal{G}=\left(G_{n}\right)_{n \in \mathbb{N}^{*}}$ be an ascending sequence of Banach Lie groups such that all inclusion maps $j_{n}: G_{n} \rightarrow G_{n+1}$ are analytic group morphisms and assume that we have the following properties:

(1) For each $n \in \mathbb{N}^{*}$, there exists a norm $\|\cdot\|_{n}$ on the Lie algebra $\mathfrak{g}_{n}$ defining its Banach space structure, such that its Lie bracket satisfies the inequality $\|[x, y]\|_{n} \leqslant\|x\|_{n}\|y\|_{n}$ for all $x$ and $y$ in $\mathfrak{g}_{n}$ and such that the bounded linear operator $\mathbb{L}\left(j_{n}\right): \mathfrak{g}_{n} \rightarrow \mathfrak{g}_{n+1}$ has a norm operator bounded by 1 ;

(2) The locally convex structure of vector space $\mathfrak{g}=\lim _{\mathfrak{g}} \mathfrak{g}_{n}$ is Hausdorff;

(3) The map $\exp _{G}=\bigcup_{n \in \mathbb{N}^{*}} \exp _{G_{n}}: \bigcup_{n \in \mathbb{N}^{*}} \mathfrak{g}_{n} \rightarrow \bigcup_{n \in \mathbb{N}^{*}} G_{n}$ is injective on some neighborhood of 0 .

Then $G=\lim _{\longrightarrow} G_{n}=\bigcup_{n \in \mathbb{N}^{*}} G_{n}$ has an analytic structure of Lie group modelled on $\mathfrak{g}$ and $\exp _{G}$ is an analytic diffeomorphism from some neighborhood of 0 to a neighborhood of $1 \in G$.

We end this subsection with an application of this result ${ }^{(6)}$ :

(6) This result is certainly well known by specialists but it is an easy corollary of Theorem 4.14 and so we give a proof here. 
THEOREM 4.15. - Let $\left(E_{n}\right)_{n \in \mathbb{N}^{*}}$ be an ascending sequence of Banach spaces such that $E_{n}$ is a complemented Banach subspace of $E_{n+1}$. Then $E=\bigcup_{n \in \mathbb{N}^{*}} E_{n}$ is an LB-space and $L(E)=\bigcup_{n \in \mathbb{N}^{*}} L\left(E_{n}\right)$ is also an LBspace, where $L\left(E_{n}\right)$ is the Banach space of continuous linear operators of $E_{n}$. Moreover, $G L(E)=\bigcup_{n \in \mathbb{N}^{*}} G L\left(E_{n}\right)$ has a structure of convenient Lie group modelled on $L(E)$, where $G L\left(E_{n}\right)$ is the Banach Lie group of linear continuous automorphisms of $E_{n}$.

For the proof of this theorem we need the following lemma:

Lemma 4.16. - Let $E$ and $F$ be two Banach spaces such that $E$ is a complemented Banach subspace of $F$. Given a norm $\|\cdot\|_{E}$ on $E$, there exists a norm $\|\cdot\|_{F}$ on $F$ and an embedding $\lambda: L(E) \rightarrow L(F)$ which is an isometry with respect to the corresponding operator norms on $L(E)$ and $L(F)$ respectively.

Moreover, we have $\left[\lambda(T), \lambda\left(T^{\prime}\right)\right]=\lambda\left(\left[T, T^{\prime}\right]\right)$ where, as classically, the bracket is given by $\left[T, T^{\prime}\right]=T \circ T^{\prime}-T^{\prime} \circ T$.

Proof. - Let $E^{\prime}$ be a subspace of $F$ such that $F=E \oplus E^{\prime}$. We endow $E^{\prime}$ with a norm $\|\cdot\|^{\prime}$ and let $\|\cdot\|_{F}$ be the norm on $F$ defined by $\|x\|_{F}=$ $\left\|x_{1}\right\|_{E}+\left\|x_{2}\right\|^{\prime}$ if $x=x_{1}+x_{2}$ with $x_{1} \in E$ and $x_{2} \in E^{\prime}$. Denote by $\lambda$ the natural inclusion of $E$ in $F$. By construction, $\lambda$ is an isometry. We define $\Lambda: L(E) \rightarrow L(F)$ where $\Lambda(T)$ is the operator on $F$ whose restriction to $E$ is $T$ and whose restriction to $E^{\prime}$ is the null operator. Clearly $\Lambda$ is injective and the operator norm of $\Lambda$ is 1 . Indeed if $\Pi$ is the projection of $F$ on $E$ with kernel $E^{\prime}$, we have

$$
\frac{\|\Lambda(T)(x)\|_{F}}{\|x\|_{F}} \leqslant \frac{\|T \circ \Pi(x)\|_{F}}{\|\Pi(x)\|_{E}} \leqslant\|T\|_{L(E)} .
$$

We deduce $\|\Lambda(T)\|_{L(F)} \leqslant\|T\|_{L(E)}$. On the other hand

$$
\begin{aligned}
\|T\|_{L(E)} & =\sup \left\{\frac{\|T(x)\|_{E}}{\|x\|_{E}}, x \in E\right\} \\
& \leqslant \sup \left\{\frac{\|T(x)\|_{E}}{\|x\|_{F}}, x \in F\right\}=\sup \left\{\frac{\|\Lambda(T)(x)\|_{F}}{\|x\|_{F}}, x \in F\right\} .
\end{aligned}
$$

Finally, it is easy to verify that we have $\Lambda\left(T \circ T^{\prime}\right)=\Lambda(T) \circ \Lambda\left(T^{\prime}\right)$, which ends the proof.

Proof of Theorem 4.15. - According to Lemma 4.16, by induction, we can build a sequence of norms $\|\cdot\|_{n}$ on each $E_{n}$ and an isometry $\Lambda_{n}$ : $L\left(E_{n}\right) \rightarrow L\left(E_{n+1}\right)$. For simplicity, we identify $L\left(E_{n}\right)$ with $\Lambda_{n}\left(L\left(E_{n}\right)\right)$ in $L\left(E_{n+1}\right)$. Then $L\left(E_{n}\right)$ is a Banach subspace of $L\left(E_{n+1}\right)$ with the induced topology. It follows that $\mathfrak{G}=\bigcup_{n \in \mathbb{N}} L\left(E_{n}\right)=\underline{\lim _{1}} L\left(E_{n}\right)$ is a convenient space. 
On the other hand, for the operator norm in each $L\left(E_{n}\right)$ we have

$$
\left\|\left[T, T^{\prime}\right]\right\|_{L\left(E_{n}\right)} \leqslant 2\|T\|_{L\left(E_{n}\right)}\left\|T^{\prime}\right\|_{L\left(E_{n}\right)}
$$

On each $L\left(E_{n}\right)$, we consider the norm $\nu_{n}=2\|\cdot\|_{L\left(E_{n}\right)}$. Then $\nu_{n}$ defines the topology of $L\left(E_{n}\right)$. The inclusion $\Lambda_{n}$ is still an isometry and we have

$$
\nu_{n}\left(\left[T, T^{\prime}\right]\right) \leqslant \nu_{n}(T) \nu_{n}\left(T^{\prime}\right) .
$$

Given $T \in \mathfrak{G}$, then $T$ belongs to some $L\left(E_{n}\right)$; we then have

$$
\exp _{\mathfrak{G}}(T)=\sum_{k \in \mathbb{N}} \frac{T^{k}}{k !}
$$

On one hand, classically, the exponential map $\exp _{n}: L\left(E_{n}\right) \rightarrow G L\left(E_{n}\right)$ is an analytic diffeomorphism over the ball $B_{n}(0, \ln (2))$ (relative to the norm $\nu_{n}$ on $\left.L\left(E_{n}\right)\right)$ in $G L\left(E_{n}\right)$. On the other hand, we have the relations: $\exp _{n+1 \mid L\left(E_{n}\right)}=\exp _{n}$ and $B_{n+1}\left(0, \frac{1}{2} \ln (2)\right) \bigcap E_{n}=B_{n}\left(0, \frac{1}{2} \ln (2)\right)$. It follows that $\exp _{\mathfrak{G}}$ is injective on $\bigcup_{n \in \mathbb{N}} B_{n}\left(0, \frac{1}{2} \ln (2)\right)$. Therefore all the assumptions of Theorem 4.14 are satisfied and we get the announced result.

\subsection{Direct limit of Banach vector bundles}

Definition 4.17. - A sequence $\left(E_{n}, \pi_{n}, M_{n}\right)_{n \in \mathbb{N}^{*}}$ of Banach vector bundles is called a strong ascending sequence of Banach vector bundles if the following assumptions are satisfied:

(A) $\mathcal{M}=\left(M_{n}\right)_{n \in \mathbb{N}^{*}}$ is an ascending sequence of Banach $C^{\infty}$-manifolds, where $M_{n}$ is modelled on the Banach space $\mathbb{M}_{n}$ such that $\mathbb{M}_{n}$ is a complemented Banach subspace of $\mathbb{M}_{n+1}$ and the inclusion $\varepsilon_{n}^{n+1}$ : $M_{n} \rightarrow M_{n+1}$ is a $C^{\infty}$ injective map such that $\left(M_{n}, \varepsilon_{n}^{n+1}\right)$ is a weak submanifold of $M_{n+1}$;

(B) The sequence $\left(E_{n}\right)_{n \in \mathbb{N}^{*}}$ is an ascending sequence such that the sequence of typical fibers $\left(\mathbb{E}_{n}\right)_{n \in \mathbb{N}^{*}}$ of $\left(E_{n}\right)_{n \in \mathbb{N}^{*}}$ is an ascending sequence of Banach spaces such that $\mathbb{E}_{n}$ is a complemented Banach subspace of $\mathbb{E}_{n+1}$;

(C) For each $n \in \mathbb{N}^{*}, \pi_{n+1} \circ \lambda_{n}^{n+1}=\varepsilon_{n}^{n+1} \circ \pi_{n}$ where $\lambda_{n}^{n+1}: E_{n} \rightarrow E_{n+1}$ is the natural inclusion;

(D) Any $x \in M=\lim _{\longrightarrow} M_{n}$ has the direct limit chart property for $(U=$ $\left.\lim _{\longrightarrow} U_{n}, \phi=\lim _{\phi_{n}}\right)$; 
(E) For each $n \in \mathbb{N}^{*}$, there exists a trivialization $\Psi_{n}:\left(\pi_{n}\right)^{-1}\left(U_{n}\right) \rightarrow$ $U_{n} \times \mathbb{E}_{n}$ such that the following diagram is commutative:

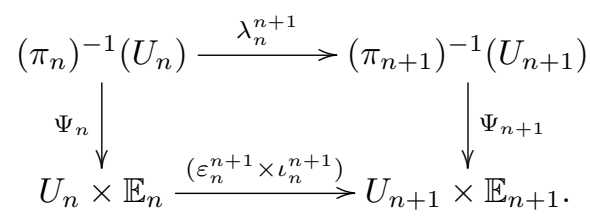

For example, the sequence $\left(T M_{n}, \pi_{n}, M_{n}\right)_{n \in \mathbb{N}^{*}}$ is a strong ascending sequence of Banach vector bundles whenever $\left(M_{n}\right)_{n \in \mathbb{N}^{*}}$ is an ascending sequence which has the direct limit chart property at each point of $x \in M=$ $\stackrel{\lim _{\longrightarrow}}{ } M_{n}$ whose model $\mathbb{M}_{n}$ is complemented in $\mathbb{M}_{n+1}$.

Proposition 4.18. - Let $\left(E_{n}, \pi_{n}, M_{n}\right)_{n \in \mathbb{N}^{*}}$ be a strong ascending sequence of Banach vector bundles. We have:

(1) $\lim _{\longrightarrow} E_{n}$ has a structure of n.n.H convenient manifold modelled on $\overrightarrow{\text { the }}$ LB-space $\lim \mathbb{M}_{n} \times \lim _{\mathbb{E}}$ which has a Hausdorff convenient structure if and only if $\vec{M}$ is Hausdorff.

(2) $\left(\lim _{\longrightarrow} E_{n}, \underset{\lim }{\longrightarrow} \pi_{n}, \lim _{\longrightarrow} M_{n}\right)$ can be endowed with a structure of conve-

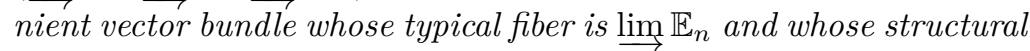
group is a metrizable complete topological group, projective limit of a sequence of Banach-Lie groups.

Proof. -

(1). - Consider $(x, v)$ in some $E_{n}$; in particular $x$ belongs to $M_{n}$. According to the assumptions (C) and (D), there exists a chart $(U, \phi)$ of $M=\lim _{i} M_{i}$ around $x$ where $\phi: U=\bigcup_{i \geqslant n} U_{i} \rightarrow V=\bigcup_{i \geqslant n} \phi_{i}\left(U_{i}\right)$,

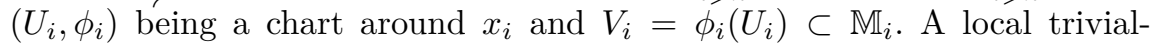
ization $\Psi_{i}: \pi_{i}^{-1}\left(U_{i}\right) \rightarrow U_{i} \times \mathbb{E}_{i}$ gives rise, via the chart $\phi_{i}: U_{i} \rightarrow V_{i}$, to a chart $\psi_{i}: \pi_{i}^{-1}\left(U_{i}\right) \rightarrow V_{i} \times \mathbb{E}_{i} \subset \mathbb{M}_{i} \times \mathbb{E}_{i}$.

From the assumption (E), we get the commutativity of the diagram

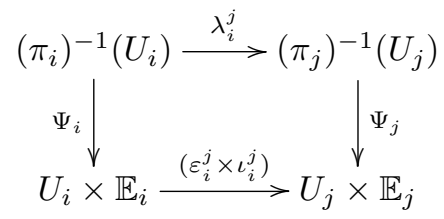

The previous arguments imply that the sequence of Banach manifolds $\left\{E_{n}\right\}_{n \in \mathbb{N}^{*}}$ has the direct limit chart property at any point $(x, v) \in \underline{\lim } E_{n}$. 
Therefore, from Theorem 4.8, there exists a unique structure of n.n.H. convenient manifold on $E=\lim _{n} E_{n}$ whose topology coincides with the $D L$ topology on $E$. In particular this structure is Hausdorff if and only if $M$ is so.

(2). - The main difficulty is to define the structural group ${ }^{(7)}$, say $G(\mathbb{E})$ where $\mathbb{E}=\lim _{\longrightarrow} \mathbb{E}_{n}$.

Let $\mathbb{E}_{1} \subset \mathbb{E}_{2} \subset \ldots$ be the direct sequence of complemented Banach spaces associated to the direct sequence $E_{1} \subset E_{2} \subset \ldots$; so there exist Banach subspaces $\mathbb{E}_{1}^{\prime}, \mathbb{E}_{2}^{\prime}, \ldots$ such that:

$$
\left\{\begin{array}{l}
\mathbb{E}_{1}=\mathbb{E}_{1}^{\prime}, \\
\forall i \in \mathbb{N}^{*}, \mathbb{E}_{i+1} \simeq \mathbb{E}_{i} \times \mathbb{E}_{i+1}^{\prime} .
\end{array}\right.
$$

For $i, j \in \mathbb{N}^{*}, i \leqslant j$, we have the injection

$$
\begin{aligned}
\iota_{i}^{j}: \mathbb{E}_{i} \simeq \mathbb{E}_{1}^{\prime} \times \cdots \times \mathbb{E}_{i}^{\prime} & \longrightarrow \mathbb{E}_{j} \simeq \mathbb{E}_{1}^{\prime} \times \cdots \times \mathbb{E}_{j}^{\prime} \\
\left(x_{1}^{\prime}, \ldots, x_{i}^{\prime}\right) & \longmapsto\left(x_{1}^{\prime}, \ldots, x_{i}^{\prime}, 0, \ldots, 0\right)
\end{aligned}
$$

Any $A_{n+1} \in G L\left(\mathbb{E}_{n+1}\right)$ is represented by $\left(\begin{array}{cc}A_{n} & B_{n+1} \\ A_{n}^{\prime} & B_{n+1}^{\prime}\end{array}\right)$ where $A_{n} \in \mathcal{L}\left(\mathbb{E}_{n}, \mathbb{E}_{n}\right)$, $A_{n}^{\prime} \in \mathcal{L}\left(\mathbb{E}_{n}, \mathbb{E}_{n+1}^{\prime}\right), B_{n+1} \in \mathcal{L}\left(\mathbb{E}_{n+1}^{\prime}, \mathbb{E}_{n}\right)$ and $B_{n+1}^{\prime} \in \mathcal{L}\left(\mathbb{E}_{n+1}^{\prime}, \mathbb{E}_{n+1}^{\prime}\right)$.

The group

$$
G L_{0}\left(\mathbb{E}_{n+1} \mid \mathbb{E}_{n}\right)=\left\{A \in G L\left(\mathbb{E}_{n+1}\right): A\left(\mathbb{E}_{n}\right)=\mathbb{E}_{n}\right\}
$$

can be identified with the Banach-Lie sub-group of operators of type $\left(\begin{array}{cc}A_{n} & B_{n+1} \\ 0 & B_{n+1}^{\prime}\end{array}\right)$ (cf. [5]).

The set

$$
G_{n}=\left\{A_{n} \in G L\left(\mathbb{E}_{n}\right): \forall k \in\{1, \ldots, n-1\}, A_{n}\left(\mathbb{E}_{k}\right)=\mathbb{E}_{k}\right\}
$$

can be endowed with a structure of Banach-Lie subgroup.

An element $A_{n}$ of $G_{n}$ can be seen as

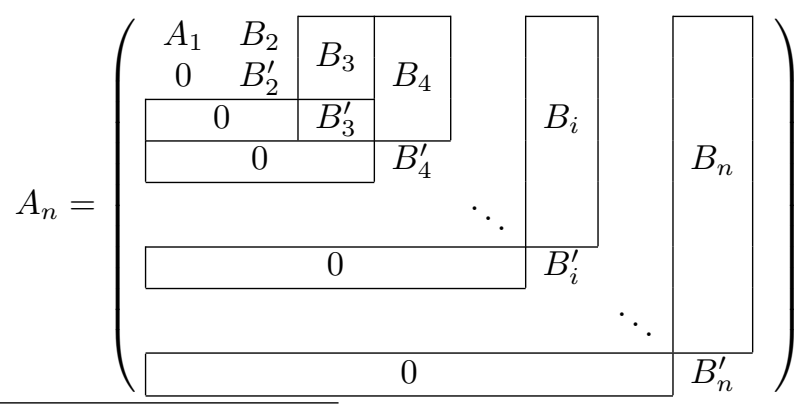

(7) As the referee pointed out, the structural group is much larger than the direct limit of the linear groups $G L\left(\mathbb{E}_{n}\right)$. 
For $1 \leqslant i \leqslant j \leqslant k$, we consider the following diagram

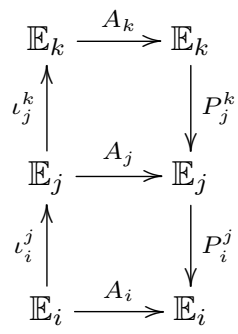

where $P_{i}^{j}: \mathbb{E}_{j} \rightarrow \mathbb{E}_{i}$ is the projection along the direction $\mathbb{E}_{i+1}^{\prime} \oplus \cdots \oplus \mathbb{E}_{j}^{\prime}$.

The map

$$
\begin{aligned}
\theta_{i}^{j}: G_{j} & \longrightarrow G_{i} \\
A_{j} & \longrightarrow P_{i}^{j} \circ A_{j} \circ \iota_{i}^{j}
\end{aligned}
$$

is perfectly defined and we have:

$$
\left(\theta_{i}^{j} \circ \theta_{j}^{k}\right)\left(A_{k}\right)=\theta_{i}^{j}\left[\theta_{j}^{k}\left(A_{k}\right)\right]=\theta_{i}^{j}\left(P_{j}^{k} \circ A_{j} \circ \iota_{j}^{k}\right)=P_{i}^{j} \circ P_{j}^{k} \circ A_{j} \circ \iota_{j}^{k} \circ \iota_{i}^{j} .
$$

Because $P_{i}^{j} \circ P_{j}^{k}=P_{i}^{k}$ (projective system) and $\iota_{j}^{k} \circ \iota_{i}^{j}=\iota_{i}^{k}$ (inductive system), we have

$$
\left(\theta_{i}^{j} \circ \theta_{j}^{k}\right)\left(A_{k}\right)=P_{i}^{k} \circ A_{j} \circ \iota_{i}^{k}=\theta_{i}^{k}\left(A_{k}\right)
$$

So $\left(G_{i}, \theta_{i}^{j}\right)_{i \leqslant j}$ is a projective system of Banach-Lie groups and the projective limit $G(\mathbb{E})=\lim G_{n}$ can be endowed with a structure of metrizable complete topological group.

From assumptions (C) and (D) it follows that we have a well defined conveniently smooth projection $\pi=\underline{\lim } \pi_{i}: \lim _{\longrightarrow} E_{i} \rightarrow \underline{\lim _{1}} M_{i}$ given by $\pi(x, v)=x$ and, with the previous notations, we also have $\underset{\lim }{\longrightarrow}\left(\pi_{i}\right)^{-1}\left(U_{i}\right)=$ $\pi^{-1}\left(\stackrel{\lim }{\longrightarrow} U_{i}\right)$.

The map $\Psi_{i}: \pi_{i}^{-1}\left(U_{i}\right) \rightarrow U_{i} \times \mathbb{E}_{i}$ can be written $\Psi_{i}\left(y_{i}, u_{i}\right)=\left(y_{i}, \tilde{\Psi}_{i}\left(y_{i}\right)\left(u_{i}\right)\right)$ where $u_{i} \mapsto \tilde{\Psi}_{i}\left(y_{i}\right)\left(u_{i}\right)$ is an isomorphism of Banach spaces from $\pi_{i}^{-1}\left(y_{i}\right)$ to $\mathbb{E}_{i}$.

Consider an atlas $\mathcal{A}=\left\{\left(U^{\alpha}=\underline{\lim _{\longrightarrow}} U_{i}^{\alpha}, \phi^{\alpha}=\underline{\lim _{i}} \phi_{i}^{\alpha}\right)\right\}_{\alpha \in A}$ on $M$. From the proof of the first part, the set $\hat{\mathcal{A}}=\left\{\left(\pi^{-1}\left(\overrightarrow{U^{\alpha}}\right)=\lim _{\longrightarrow} \pi_{i}^{-1}\left(U_{i}^{\alpha}\right)\right.\right.$, $\left.\left.\psi^{\alpha}=\lim _{\longrightarrow} \psi_{i}^{\alpha}\right)\right\}_{\alpha \in A}$ is an atlas for the manifold $E$.

Now, if $U_{i}^{\alpha} \cap U_{i}^{\beta} \neq \emptyset$, $\Psi_{i}^{\alpha} \circ\left(\Psi_{i}^{\beta}\right)^{-1}: \phi_{i}^{\beta}\left(U_{i}^{\alpha} \cap U_{i}^{\beta}\right) \times \mathbb{E}_{1}^{\prime} \times \cdots \times \mathbb{E}_{i}^{\prime} \longrightarrow \phi_{i}^{\alpha}\left(U_{i}^{\alpha} \cap U_{i}^{\beta}\right) \times \mathbb{E}_{1}^{\prime} \times \cdots \times \mathbb{E}_{i}^{\prime}$ 
can be written

$$
\left(\bar{y}_{i}^{\beta},{\overline{u^{\prime}}}_{1}^{\beta}, \ldots,{\overline{u^{\prime}}}_{i}^{\beta}\right) \mapsto\left(\phi_{i}^{\alpha} \circ\left(\phi_{i}^{\beta}\right)^{-1}\left(\bar{y}_{i}^{\beta}\right),\left[\tilde{\Psi}_{i}^{\alpha}\left(y_{i}\right)\right] \circ\left[\tilde{\Psi}_{i}^{\beta}\left(y_{i}\right)\right]^{-1}\left({\overline{u^{\prime}}}_{1}^{\beta}, \ldots,{\overline{u^{\prime}}}_{i}^{\beta}\right)\right)
$$

where $\bar{y}_{i}^{\beta}=\phi_{i}^{\beta}\left(y_{i}\right)$. With these notations, $\bar{y}_{i}^{\beta} \mapsto \Theta_{i}^{\alpha \beta}\left(\bar{y}_{i}^{\beta}\right)=\left[\tilde{\Psi}_{i}^{\alpha}\left(y_{i}\right)\right] \circ$ $\left[\tilde{\Psi}_{i}^{\beta}\left(y_{i}\right)\right]^{-1}$ is a conveniently smooth map.

From assumption (C) and assumption (E) written over the open sets $U_{i}^{\alpha}$ and $U_{j}^{\alpha}$ (resp. $U_{i}^{\beta}$ and $U_{j}^{\beta}$ ), we have

$$
\left(\tilde{\Psi}_{j}^{\alpha}\left(y_{j}\right)\right)^{-1} \circ \iota_{i}^{j}=\lambda_{i}^{j} \circ\left(\tilde{\Psi}_{i}^{\alpha}\left(y_{i}\right)\right)^{-1} .
$$

Finally we get

$$
\Theta_{j}^{\alpha \beta}\left(\bar{y}_{j}\right) \circ \iota_{i}^{j}=\iota_{i}^{j} \circ \Theta_{i}^{\alpha \beta}\left(\bar{y}_{i}\right)
$$

So if $\bar{y}=\underset{\lim }{\longrightarrow} \bar{y}_{i}$, from the above relation, one can define the transition function $\Theta^{\alpha \beta}(\bar{y})$ as an element of the metrizable complete topological group $G(\mathbb{E})$.

\section{Linear connections on direct limit of anchored Banach bundles}

\subsection{Bundle structures on the tangent bundle to a vector bundle}

Let $M$ be a smooth Banach manifold modelled on a Banach space $\mathbb{M}$ and let $\pi: E \rightarrow M$ be a smooth Banach vector bundle on $M$ whose typical fiber is a Banach space $\mathbb{E}$. Let $p_{E}: T E \rightarrow E$ and $p_{M}: T M \rightarrow M$ be the canonical projections of each tangent bundle.

There exists an atlas $\left\{U^{\alpha}, \phi^{\alpha}\right\}_{\alpha \in A}$ of $M$ for which $E_{\mid U^{\alpha}}$ is trivial; therefore we obtain a chart $\left(U_{E}^{\alpha}, \phi_{E}^{\alpha}\right)$ on $E$, where $U_{E}^{\alpha}=\pi^{-1}\left(U^{\alpha}\right)$ and s.t. $\phi_{E}^{\alpha}$ is a diffeomorphism from $U_{E}^{\alpha}$ on $\phi^{\alpha}\left(U^{\alpha}\right) \times \mathbb{E}$. We also have a chart $\left(U_{T M}^{\alpha}, \phi_{T M}^{\alpha}\right)$ on $T M$ where $U_{T M}^{\alpha}=p_{M}^{-1}\left(U^{\alpha}\right)$ and $\phi_{T M}^{\alpha}=\left(\phi^{\alpha}, T p_{M}\right)$.

Hence the family $\left\{T\left(E_{\mid U^{\alpha}}\right), T \phi_{E}^{\alpha}\right\}_{\alpha \in A}$ where

$$
T \phi_{E}^{\alpha}: T\left(E_{\mid U^{\alpha}}\right) \longrightarrow T\left(\phi^{\alpha}\left(U^{\alpha}\right) \times \mathbb{E}\right)=\phi^{\alpha}\left(U^{\alpha}\right) \times \mathbb{E} \times \mathbb{M} \times \mathbb{E}
$$

is the atlas describing the canonical vector bundle structure of $\left(T E, p_{E}, E\right)$.

Let $(x, u)$ be an element of $E_{x}=\pi^{-1}(x)$ where $x \in U^{\alpha \beta}=U^{\alpha} \cap$ $U^{\beta} \neq \emptyset$ and let $(x, u, y, v)$ be an element of $T_{(x, u)} E$. For $\left(x^{\alpha}, u^{\alpha}, y^{\alpha}, v^{\alpha}\right)=$ $T \phi_{E}^{\alpha}(x, u, y, v)$, we have the transition functions:

$$
\begin{aligned}
& \left(T\left(\left(\phi^{\alpha} \times \operatorname{Id}_{\mathbb{E}}\right) \circ \phi_{E}^{\alpha}\right) \circ\left(T\left(\left(\phi^{\beta} \times \operatorname{Id}_{\mathbb{E}}\right) \circ \phi_{E}^{\beta}\right)\right)^{-1}\right)\left(\left(x^{\beta}, u^{\beta}, y^{\beta}, v^{\beta}\right)\right) \\
& =\left(\phi^{\alpha \beta}\left(x^{\beta}\right), \phi_{E}^{\alpha \beta}\left(\left(\phi^{\beta}\right)^{-1}\left(y^{\beta}\right)\right) u^{\beta}, d \phi^{\alpha \beta}\left(x^{\beta}\right) y^{\beta},\left(d\left(\phi_{E}^{\alpha \beta} \circ\left(\phi^{\beta}\right)^{-1}\right)\left(x^{\beta}\right) y^{\beta}\right) u^{\beta}\right) \\
& +\phi_{E}^{\alpha \beta}\left(\left(\phi^{\beta}\right)^{-1}\left(x^{\beta}\right) v^{\beta}\right)
\end{aligned}
$$


where $\phi^{\alpha \beta}=\phi^{\alpha} \circ\left(\phi^{\beta}\right)^{-1}$ and $\left(x^{\beta}, \phi_{E}^{\alpha \beta}\left(x^{\beta}\right) u^{\beta}\right)=\left(\phi_{E}^{\alpha} \circ\left(\phi_{E}^{\beta}\right)^{-1}\right)\left(x^{\beta}, u^{\beta}\right)$ for $x^{\beta} \in \phi^{\alpha}\left(U^{\alpha \beta}\right)$.

So, for fixed $\left(x^{\beta}, u^{\beta}\right)$, the transition functions are linear in $\left(y^{\beta}, v^{\beta}\right) \in$ $\mathbb{M} \times \mathbb{E}$. This describes the vector bundle structure of the tangent bundle $\left(T E, p_{E}, E\right)$.

On the other hand, for fixed $\left(x^{\beta}, y^{\beta}\right)$ the transition functions of TE are also linear in $\left(u^{\beta}, v^{\beta}\right) \in \mathbb{E} \times \mathbb{E}$ and we get a vector bundle structure on (TE, T $\pi, T M)$ which appears as the derivative of the original one on $E$.

\subsection{Connections on a Banach bundle}

The kernel of $T \pi: T E \rightarrow T M$ is denoted by $V E$ and is called the vertical bundle over $E$. It appears as a vector bundle over $M$. It is well known that $V E$ can also be seen as the pull-back of the bundle $\pi: E \rightarrow M$ over $\pi$ as described by the following diagram:

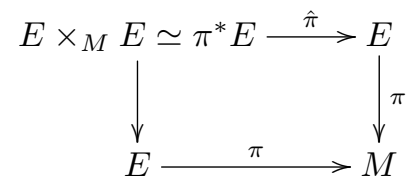

We have a canonical isomorphism $E \times_{M} E \rightarrow V E$ called the vertical lift $v l_{E}$ defined by

$$
v l_{E}(x, u, v)=\dot{\gamma}(0)
$$

where $\gamma(t)=u+t v$. This map is fiber linear over $M$.

Let $J: V E \rightarrow T E$ be the canonical inclusion. According to [32] we have:

Definition 5.1. - A (non linear) connection on $E$ is a bundle morphism $V: T E \rightarrow V E$ such that $V \circ J=\mathrm{Id}_{V E}$.

The datum of a connection $V$ on $E$ is equivalent to the existence of a decomposition $T E=H E \oplus V E$ of the Banach bundle $E$ with $H E=\operatorname{ker} V$.

We then have the following diagram:

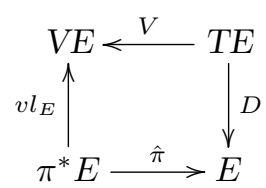

The bundle morphism $D=\hat{\pi} \circ v l_{E}^{-1} \circ V: T E \rightarrow E$ is called the connection map or connector which is a smooth morphism of fibrations. Note that, in 
each fiber $T_{(x, u)} E$, the kernel of $D$ is exactly the subspace $H_{(x, u)} E$ of $H E$ in $T_{(x, u)} E$. Therefore, the datum of $D$ is equivalent to the datum of $V$.

We then have, modulo the identification $V E \simeq \pi^{*} E$ via $v l_{E}$ :

$$
\begin{aligned}
D: T E & \longrightarrow E \\
(x, u, y, v) & \longmapsto(x, v+\omega(x, u) y)
\end{aligned}
$$

where $\omega(x, u) \in L\left(T_{x} M, T_{(x, u)} E\right)$.

If moreover, $D$ is linear on each fiber, then the connection is called a linear connection.

Modulo the identification of $U \subset M$ and $\phi(U) \subset \mathbb{M}$ we have the following identifications:

- $E_{\mid U} \equiv U \times \mathbb{E}$

- $T M_{\mid U} \equiv U \times \mathbb{M}$

- $T E_{\mid \pi^{-1}(U)} \equiv(U \times \mathbb{E}) \times(\mathbb{M} \times \mathbb{E})$

- $V E_{\mid \pi^{-1}(U)} \equiv(U \times \mathbb{E}) \times \mathbb{E}$

According to these identifications, we obtain the following characterizations of $V$ and $D$ :

$$
\begin{aligned}
& V(x, u, y, v)=(x, u, 0, v+\omega(x, u) y) \\
& D(x, u, y, v)=(x, v+\omega(x, u) y)
\end{aligned}
$$

where $\omega$ is a smooth map from $U \times \mathbb{E}$ to the space $L(\mathbb{M}, \mathbb{E})$ of bounded linear operators from $\mathbb{M}$ to $\mathbb{E}$.

This connection is linear if and only if $\omega$ is linear in the second variable. In this case, the relation $\Gamma(x)(u, y)=\omega(x, u) y$ gives rise to a smooth map $\Gamma$ from $U$ to the space of bilinear maps $L^{2}(\mathbb{E}, \mathbb{M} ; \mathbb{E})$ called local Christoffel components of the connection.

Conversely, a connection can be given by a collection $\left(U^{\alpha}, \omega^{\alpha}\right)$ of local maps $\omega^{\alpha}: U^{\alpha} \times \mathbb{E} \rightarrow L(\mathbb{M}, \mathbb{E})$ on a covering $\left(U^{\alpha}\right)$ of $M$ with adequate classical conditions of compatibility between $\left(U^{\alpha}, \omega^{\alpha}\right)$ and $\left(U^{\beta}, \omega^{\beta}\right)$ where $U_{a} \cap U_{\beta} \neq \emptyset$.

Remark 5.2. - It is classical that if $M$ is smooth paracompact, then there always exists a connection on $M$ and also on each Banach bundle over $M$. However, these assumptions impose the same assumptions on the Banach space $\mathbb{M}$.

On the other hand, it is well known that there exist linear connections on a Banach manifold without such assumptions. For instance, if $T M \equiv M \times \mathbb{M}$ there always exists a (trivial) connection on $M$. But there are further situations for which a linear connection exists on a Banach manifold. For example, 
there exist linear connections on loop spaces (see for instance [7]) or on the manifold $\mathcal{M}(\mu)$ of strictly positive probability densities of a probability space $(\Omega, \Sigma, \mu)$ (cf. [24]).

Definition 5.3. - A Koszul connection on $E$ is a $\mathbb{R}$-bilinear map $\nabla$ : $\mathfrak{X}(M) \times \underline{E} \rightarrow \underline{E}$ which fulfills the following properties:

(i) $\nabla_{X}(f \sigma)=\mathrm{d} f(X) \sigma+f \nabla_{X} \sigma$

(ii) $\nabla_{f X} \sigma=f \nabla_{X} \sigma$

for any function $f$ on $M, X \in \chi(M)$ and $\sigma \in \underline{E}$.

Given a linear connection $D$ on a Banach bundle $\pi: E \rightarrow M$, we obtain a covariant derivative $\nabla: \mathfrak{X}(M) \times \underline{E} \rightarrow \underline{E}$ which is a Koszul connection. Since any (linear) connection induces naturally a (linear) connection on the restriction $E_{\mid U}$ of $E$ to any open set $U$ of $M$, we also obtain a covariant derivative $\nabla^{U}: \mathfrak{X}(U) \times E_{\mid U} \rightarrow E_{\mid U}$ with the correspondent previous properties for any function $f$ on $\bar{U}, X \overline{\in \mathcal{X}}(U)$ and $\sigma \in \underline{E_{\mid U}}$.

Unfortunately, in general, a Koszul connection may be not localizable in the following sense: since any local section of $E$ (resp. any local vector field on $M$ ) can not be always extended to a global section of $E$ (resp. to a global vector field on $M$ ), the previous operator $\nabla$ can not always induce a (local) operator $\nabla^{U}$ as previously. Therefore, in this work, a Koszul connection will always be taken in the sense of the covariant derivative associated to a linear connection $D$ on $E$. In particular, for any $x \in M$, the value $\nabla_{X} \sigma(x)$ only depends on of the value of $X$ at $x$ and the 1-jet of $\sigma$ at $x$.

In a local trivialization $E_{\mid U} \equiv U \times \mathbb{E}$, a local section $\sigma$ of $E$, defined on $U$, can be identified with a map $\sigma: U \rightarrow \mathbb{E}$. Then $\nabla$ has the local expression:

$$
\nabla_{X} \sigma=\mathrm{d} \sigma(X)+\Gamma(\sigma, X)
$$

where $\Gamma$, smooth map from $U$ to $L^{2}(\mathbb{E}, \mathbb{M} ; \mathbb{E})$, is the local Christoffel components of the connection $D$ which will be also called the local Christoffel components of $\nabla$.

Remark 5.4. - If $M$ is smooth regular, then, as classically in finite dimension, any covariant derivative $\nabla: \mathfrak{X}(M) \times \underline{E} \rightarrow \underline{E}$ which fulfills the previous properties (i) and (ii) is localizable. Therefore, in this case, there is a one-to-one correspondence between such covariant derivative and linear connection on $E$ as in the finite dimensional framework.

Finally if $E_{\mid U} \equiv U \times \mathbb{E}$ and $E_{\mid U^{\prime}} \equiv U^{\prime} \times \mathbb{E}$ are local trivializations such that $U \cap U^{\prime} \neq \emptyset$, then we have a smooth map $g: U \cap U^{\prime} \rightarrow G L(\mathbb{E})$ such 
that $\sigma_{\mid U^{\prime}}=g \sigma_{\mid U}$ for any section defined on $U \cup U^{\prime}$. Therefore the Christoffel component $\Gamma$ and $\Gamma^{\prime}$ of $\nabla$ on $U \cap U^{\prime}$ are linked by the relation

$$
\Gamma^{\prime}(X, \sigma)=g^{-1} \mathrm{~d} g(X, \sigma)+g^{-1} \Gamma(X, g \sigma) .
$$

\subsection{Direct limit of Banach connections}

DEFINITION 5.5. - Let $\left(E_{n}, \pi_{n}, M_{n}\right)_{n \in \mathbb{N}^{*}}$ be a strong ascending sequence of Banach vector bundles where $\varepsilon_{n}^{n+1}: M_{n} \rightarrow M_{n+1}$ and $\lambda_{n}^{n+1}: E_{n} \rightarrow E_{n+1}$ are the compatible bonding maps. A sequence of connections $D_{n}: T E_{n} \rightarrow E_{n}$ is called a strong ascending sequence of Banach connections if

$$
\lambda_{n}^{n+1} \circ D_{n}=D_{n+1} \circ T \lambda_{n}^{n+1} .
$$

THEOREM 5.6. - Let $\left(D_{n}\right)_{n \in \mathbb{N}^{*}}$ be a strong ascending sequence of Banach connections on an ascending sequence $\left(E_{n}, \pi_{n}, M_{n}\right)_{n \in \mathbb{N}^{*}}$ of Banach bundles and assume that $\left(M_{n}\right)_{n \in \mathbb{N}^{*}}$ has the direct limit chart property at each point of $x \in M=\underline{\lim } M_{n}$.

Then the direct limit $D=\lim _{\longrightarrow} D_{n}$ is a connection on the convenient vector bundle $\left(\stackrel{\lim }{\longrightarrow} E_{n}, \stackrel{\lim }{\longrightarrow} \pi_{n}, \stackrel{\lim }{\longrightarrow} M_{n}\right)$.

Proof. - Let $x$ be in $\lim _{n} M_{n}$. We suppose that $x \in M_{n_{0}}$. According to Definition 4.17 , let $\left(U^{\alpha}, \overrightarrow{\phi^{\alpha}}\right)$ be a chart of $M=\lim M_{n}$ around $x$ which satisfies the assumption (D) and (E), where $\phi^{\alpha}: \overrightarrow{U^{\alpha}}=\bigcup_{i \geqslant n_{0}} U_{i}^{\alpha} \rightarrow O^{\alpha}=$ $\bigcup_{i \geqslant n_{0}} O_{i}^{\alpha}$ with $\left(U_{i}^{\alpha}, \phi_{i}^{\alpha}\right)$ is a chart around $x_{i}$ and $O_{i}^{\alpha}=\phi_{i}^{\alpha}\left(U_{i}^{\alpha}\right) \subset \mathbb{M}_{i}$. Moreover, $E_{i \mid U_{i}}$ is trivial.

Denote by $D_{i}^{\alpha}$ the expression of the connection $D_{i}$ in local charts. We then have $D_{i}^{\alpha}\left(x_{i}^{\alpha}, u_{i}^{\alpha}, y_{i}^{\alpha}, v_{i}^{\alpha}\right)=\left(x_{i}^{\alpha}, v_{i}^{\alpha}+\omega_{i}^{\alpha}\left(x_{i}^{\alpha}, u_{i}^{\alpha}\right) y_{i}^{\alpha}\right)$ where $\omega_{i}^{\alpha}$ is a smooth map from $O_{i}^{\alpha} \times \mathbb{E}_{i}$ to the space $L\left(\mathbb{M}_{i}, \mathbb{E}_{i}\right)$ of bounded linear operators from $\mathbb{M}_{i}$ to $\mathbb{E}_{i}$.

Using the relations $\lambda_{i}^{i+1} \circ D_{i}=D_{i+1} \circ T \lambda_{i}^{i+1}$ we have the following diagram:

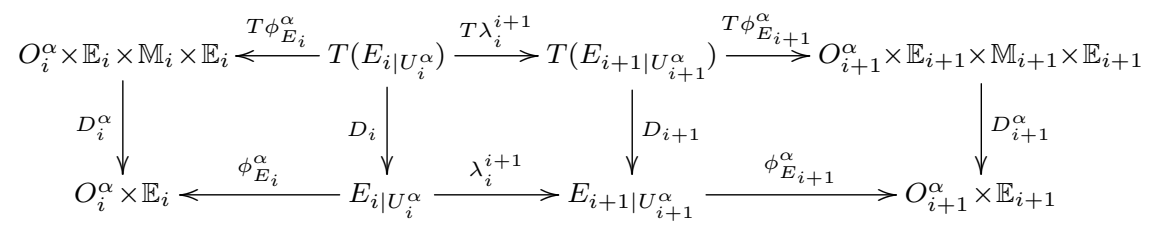

Using the expression in local coordinates ${\widehat{\varepsilon_{i}^{i+1}}}^{\alpha}: O_{i}^{\alpha} \rightarrow O_{i+1}^{\alpha}$ and the map $\widehat{\lambda_{i}^{i+1}}: \mathbb{E}_{i} \rightarrow \mathbb{E}_{i+1}$ we then obtain that $\left(D_{i}^{\alpha}\right)_{i \geqslant n}$ can be realized as a 
direct limit because we have:

$$
\begin{aligned}
\left(\widehat{\varepsilon}_{i}^{i+1}{ }^{\alpha} \times \widehat{\lambda_{i}^{i+1}}\right) \circ D_{i}^{\alpha} & =\left(\widehat{\varepsilon_{i}^{i+1}} \alpha \widehat{\lambda_{i}^{i+1}}\right) \circ\left(\phi_{E_{i}}^{\alpha} \circ D_{i} \circ\left(T \phi_{E_{i}}^{\alpha}\right)^{-1}\right) \\
& =\phi_{E_{i+1}}^{\alpha} \circ \underline{\lambda_{i}^{i+1} \circ D_{i}} \circ\left(T \phi_{E_{i}}^{\alpha}\right)^{-1} \\
& =\phi_{E_{i+1}}^{\alpha} \circ \frac{D_{i+1} \circ T \lambda_{i}^{i+1} \circ\left(T \phi_{E_{i}}^{\alpha}\right)^{-1}}{} \\
& =\phi_{E_{i+1}}^{\alpha} \circ\left(\phi_{E_{i+1}}^{\alpha}\right)^{-1} \circ D_{i+1}^{\alpha} \circ T \phi_{E_{i+1}}^{\alpha} \circ T \lambda_{i}^{i+1} \circ\left(T \phi_{E_{i}}^{\alpha}\right)^{-1} \\
& =D_{i+1}^{\alpha} \circ\left(\widehat{\varepsilon_{i}^{i+1}} \times \widehat{\lambda_{i}^{i+1}} \times \widehat{\varepsilon_{i}^{i+1}} \times \widehat{\lambda_{i}^{i+1}}\right)
\end{aligned}
$$

We obtain an analogous result for the smooth Banach local forms $\omega_{i}^{\alpha}: U_{i}^{\alpha} \times \mathbb{E}_{i} \rightarrow L\left(\mathbb{M}_{i}, \mathbb{E}_{i}\right)$.

Using the intrinsic link between a connection $D$ and a Koszul connection $\nabla$ we get the following result:

Corollary 5.7. - Let $\left(D_{n}\right)_{n \in \mathbb{N}^{*}}$ be a strong ascending sequence of $B a$ nach connections on a direct sequence $\left(E_{n}, \pi_{n}, M_{n}\right)_{n \in \mathbb{N}^{*}}$ of Banach bundles and consider the associated Koszul connections $\left(\nabla_{n}\right)_{n \in \mathbb{N}^{*}}$. The direct limit $\nabla=\lim _{n} \nabla_{n}$ is a Koszul connection on the convenient vector bundle $\left(\underline{\lim } E_{n}, \lim _{n}, \stackrel{\lim }{\longrightarrow} M_{n}\right)$.

Example 5.8. - Denote by $L_{l o c}^{p}\left(\mathbb{R}^{m}\right)$ the space of locally $L^{p}$ functions on $\mathbb{R}^{m}(1 \leqslant p<+\infty)$. A function belongs to $L_{\text {loc }}^{p}\left(\mathbb{R}^{m}\right)$ if and only if its restriction to any compact set $K$ of $\mathbb{R}^{m}$ belongs to $L^{p}(K)$. Since $\mathbb{R}^{m}$ is an ascending sequence of compact sets $K_{n}^{m}$ (where $K_{n}^{m} \subset \underset{K_{n+1}^{m}}{m}$ ), we have

$$
L_{l o c}^{p}\left(\mathbb{R}^{m}\right)=\lim _{\longrightarrow} L^{p}\left(K_{n}^{m}\right) .
$$

Moreover, the closure $\widehat{K_{n}^{m}}$ of the open set $K_{n+1}^{m} \backslash K_{n}^{m}$ is also compact and we have $L^{p}\left(K_{n+1}^{m}\right)=L^{p}\left(K_{n}^{m}\right) \oplus L^{p}\left(\widehat{K_{n}^{m}}\right)$. Therefore the sequence of Banach spaces $\left(L^{p}\left(K_{n}^{m}\right)\right)_{n \in \mathbb{N}^{*}}$ is an ascending sequence of complemented Banach spaces. Since the tangent bundle to each $L^{p}\left(K_{n}^{m}\right)$ is trivial, there exists a (trivial) Koszul connection on this Banach bundle. Therefore we get a Koszul connection on $L_{l o c}^{p}\left(\mathbb{R}^{m}\right)$.

Example 5.9. - Let $\left(H_{n}\right)_{n \in \mathbb{N}^{*}}$ be a sequence of Banach Lie groups and consider the Banach Lie group of cartesian products $G_{n}=\prod_{k=1}^{n} H_{k}$. The weak direct product $\prod_{k \in N^{*}}^{*} H_{k}$ is the set of all sequences $\left(h_{n}\right)_{n \in \mathbb{N}^{*}}$ such that $h_{n}=1$ for all but finitely many $n$. The weak direct product is a topological group for the box topology (see $[14,4]$ ). In fact, this weak direct product has a structure of Lie group modelled on the locally convex topological space $\bigoplus_{k \in \mathbb{N}^{*}} \mathfrak{H}_{k}$ where $\mathfrak{H}_{k}$ is the Lie algebra of $H_{k}$. The tangent space $T G_{n}$ is the vector bundle $G_{n} \times \bigoplus_{k=1}^{n} \mathfrak{H}_{k}$. Moreover, $T G_{n}$ is a complemented subbundle of $T G_{n+1}$ and is naturally endowed with the (trivial) Koszul connection. 


\subsection{Sprays on an anchored Banach bundle}

We begin this subsection with a brief presentation of the theory of semisprays on a Banach anchored bundle according to [2].

Let $\pi: E \rightarrow M$ be a Banach vector bundle on a Banach manifold modelled on a Banach space $\mathbb{M}$ whose fiber is modelled on a Banach space $\mathbb{E}$.

Definition 5.10. - A morphism of vector bundles $\rho: E \rightarrow T M$ is called an anchor. $(E, \pi, M, \rho)$ is then called a Banach anchored bundle.

Definition 5.11. - A semi-spray on an anchored bundle is a vector field $S$ on $E$ such that $T \pi \circ S=\rho$.

This means that, in a local trivialization $E_{\mid U} \equiv U \times \mathbb{E}$, we have $T \pi(S(x, u))=\rho(x) u$ for all $(x, u) \in E_{\mid U}$.

A smooth curve $c: I \subset \mathbb{R} \rightarrow E$ is called admissible if the tangent vector $\gamma^{\prime}(t)$ of $\gamma=\pi \circ c$ is precisely $\rho(c(t))$.

From [2], we have the following characterization of a semi-spray:

THEOREM 5.12. - A vector field $S$ on $E$ is a semi-spray if and only if each integral curve of $S$ is an admissible curve.

In a local trivialization $E_{\mid U} \equiv U \times \mathbf{E}$, a semi-spray can be written as

$$
S(x, u)=(x, u, \rho(x) u,-2 G(x, u)) .
$$

The Euler field $C$ is the global vector field on $E$ which is tangent to the fiber of $\pi$ (i.e. vertical) and such that the flow of $C$ is an infinitesimal homothety on each fiber. A semi-spray $S$ is called a spray if $S$ is invariant by the flow of $C$. This condition is equivalent to the nullity of the Lie bracket $[C, S]$. In this case, in a local trivialization, the function $G$ in Definition 5.11 is linear in the second variable.

Conversely, a spray can be given by a collection $\left(U^{\alpha}, G^{\alpha}\right)$ of local maps $G^{\alpha}: U^{\alpha} \times \mathbb{E} \rightarrow L(\mathbb{E}, \mathbb{E})$ on a covering $U^{\alpha}$ of $M$ with adequate classical conditions of compatibility between $\left(U^{\alpha}, G^{\alpha}\right)$ and $\left(U^{\beta}, G^{\beta}\right)$ when $U^{\alpha} \cap U^{\beta} \neq$ $\emptyset$ (cf. [2]).

Given a Koszul connection $\nabla$ on $E$ and an admissible curve $c: I \rightarrow E$ as in the infinite dimensional case, we associate an operator of differentiation $\nabla^{c}$ of the set of sections of $E$ along $\gamma=\pi \circ c$ given by $\nabla^{c} \sigma=\nabla_{\dot{\gamma}} \sigma$. In particular $c$ is a section along $\gamma$.

DeFINITION 5.13. - An admissible curve $c$ is called a geodesic of $\nabla$ if $\nabla_{\dot{\gamma}} c=\nabla^{c} c \equiv 0$. 
In a local trivialization $E_{\mid U} \equiv V \times \mathbf{E}$, an admissible curve $c: I \rightarrow E_{\mid U}$ is a geodesic of $\nabla$ if and only $\pi \circ c$ is a solution of the following differential equation:

$$
\left\{\begin{array}{l}
\dot{x}=\rho(x) u \\
\dot{u}=\Gamma(x)(u, \dot{x})
\end{array}\right.
$$

where $\Gamma$ is the local Christoffel component of $\nabla$ on $E_{\mid U}$. Therefore, if we set $G(x, u)=-\frac{1}{2} \Gamma(x)(u, \rho(x) u)$, we get a vector field $S_{U}$ on $E_{\mid U}$ which satisfies the relation given in Definition 5.11 and so is a spray on $E_{\mid U}$. Now, according to the compatibility conditions between the local Christoffel components, we obtain a unique global spray associated to $\nabla$. Conversely, as in the case of $E=T M$ (cf. [32]), given a spray $S$ on $E$, we can associate a unique connection $\nabla$ whose associated spray is $S$.

Taking into account the classical theorem of existence of a local flow of a vector field on a Banach manifold, we obtain:

TheOREM 5.14. - Let $(E, M, \rho)$ be a Banach anchored bundle. There exists a spray on $E$ if and only there exists a Koszul connection $\nabla$ on $E$. Moreover, there exists a canonical correspondence one-to-one between sprays and Koszul connections on $E$ so that an admissible curve is a geodesic of the Koszul connection $\nabla$ if and only if this curve is an integral curve of the unique $S$ associated to $\nabla$.

When $E=T M$, there exists an exponential map $\operatorname{Exp}: \mathcal{U} \subset T M \rightarrow M$, defined on an open neighborhood $\mathcal{U}$ of the zero section, such that $p_{M \mid \mathcal{U}}: \mathcal{U} \rightarrow$ $M$ is a fibration whose each fiber $\mathcal{U}_{x}$ is a star-shaped open neighborhood of 0 in $T_{x} M$. Moreover, the differential of the restriction $\operatorname{Exp}_{x}$ of $\operatorname{Exp}$ to $\mathcal{U}_{x}$ is equal to $\operatorname{Id}_{T_{x} M}$ at 0 . In particular, $\operatorname{Exp}_{x}$ is a diffeomorphism of a star-shaped open neighborhood of $0 \in T_{x} M$ onto an open neighborhood of $x \in M$.

\section{Direct limits of sequences of almost Banach Lie algebroids}

\subsection{Almost Banach Lie algebroids}

Let $(E, \pi, M, \rho)$ be a Banach anchored bundle.

If $\underline{E}$ denotes the $C^{\infty}(M)$-module of smooth sections of $E$, the morphism $\rho$ gives rise to a $C^{\infty}(M)$-module morphism $\rho: \underline{E} \rightarrow \underline{T M}=\mathfrak{X}(M)$ defined for every $x \in M$ and every section $s$ of $E$ by: $(\underline{\rho}(s))(x)=\rho(s(x))$ and still denoted by $\rho$. 
Definition 6.1. - An almost Lie bracket on an anchored bundle $(E, \pi, M, \rho)$ is a bilinear map $[\cdot, \cdot]_{E}: \underline{E} \times \underline{E}: \rightarrow \underline{E}$ which satisfies the following properties:

(1) $[\cdot, \cdot]_{E}$ is antisymmetric;

(2) (Leibniz property)

$$
\forall s_{1}, s_{2} \in \underline{E}, \forall f \in C^{\infty}(M),\left[s_{1}, f s_{2}\right]_{E}=f .\left[s_{1}, s_{2}\right]+\mathrm{d} f\left(\rho\left(s_{1}\right)\right) . s_{2} .
$$

Definition 6.2. - A Lie bracket is an almost Lie bracket whose jacobiator vanishes:

$$
\forall s_{1}, s_{2}, s_{3} \in \underline{E},\left[s_{1},\left[\left[s_{2}, s_{3}\right]\right]+\left[s_{2},\left[\left[s_{3}, s_{1}\right]\right]+\left[s_{3},\left[\left[s_{1}, s_{2}\right]\right]=0\right.\right.\right.
$$

DeFINITION 6.3. - An almost Banach Lie algebroid is an anchored bundle $(E, \pi, M, \rho)$ provided with an almost Lie bracket $[\cdot, \cdot]_{E}$. When $[\cdot, \cdot]_{E}$ is in fact a Lie bracket the associated structure $\left(E, M, \rho,[\cdot, \cdot]_{E}\right)$ is called a Banach Lie algebroid.

If $\left(E, \pi, M, \rho[\cdot, \cdot]_{E}\right)$ is a Banach Lie algebroid, $\rho: \underline{E} \rightarrow \mathfrak{X}(M)$ is a Lie algebra morphism; in particular, we have $\left[\rho s_{1}, \rho s_{2}\right]=\rho\left(\left[s_{1}, s_{2}\right]_{E}\right)$.

Notice that the converse is not true in general (take $\rho \equiv 0$ for instance).

Definition 6.4. - When we have $\left[\rho s_{1}, \rho s_{2}\right]=\rho\left(\left[s_{1}, s_{2}\right]_{E}\right)$ for all sections $s_{1}, s_{2} \in \underline{E}$, we will say that $\rho$ is a Lie morphism. In this case $\left.(E, \pi, M, \rho),[\cdot, \cdot]_{E}\right)$ is called an algebroid.

In general the almost Lie bracket of an algebroid $(E, \pi, M, \rho,[\cdot, \cdot])$ does not satisfy the Jacobi identity.

Remark 6.5. - Since the terminology of almost Poisson bracket seems generally adopted in the most recent papers on nonholonomic mechanics, in this work we have adopted the definition of an almost Lie algebroid given in [23]. Therefore taking into account the relation between almost Linear Poisson bracket and almost Lie bracket, this terminology seems to us well adapted. Therefore and according to [28], we use the denomination "algebroid" for an almost algebroid such that the anchor is a morphism of Lie algebras. Note that in [28] or in [15] an almost Lie algebroid corresponds to the previous definition of an algebroid and our denomination "almost algebroid" corresponds to "quasi-Lie algebroid" in [28] or in [15].

Example 6.6. - Consider a smooth right action $\psi: M \times G \rightarrow M$ of a connected Lie group $G$ on a Banach manifold $M$. Denote by $\mathcal{G}$ the Lie algebra of $G$. We then have a natural morphism $\xi$ of Lie algebras from $\mathcal{G}$ to $\mathfrak{X}(M)$ defined by:

$$
\begin{aligned}
\xi_{X}(x) & =T_{(x, e)} \psi(0, X) . \\
& -944-
\end{aligned}
$$


For any $X$ and $Y$ in $\mathcal{G}$, we have: $\xi_{\{X, Y\}}=\left[\xi_{X}, \xi_{Y}\right]$ where $\{\cdot, \cdot\}$ denotes the bracket on the Lie algebra $\mathcal{G}$ (see for instance [21, 36.12]).

On the trivial bundle $M \times \mathcal{G}$, each section can be identified with a map $\sigma$ : $M \rightarrow \mathcal{G}$. We then define a Lie bracket $\{\{\cdot, \cdot\}\}$ on the set of such sections by:

$$
\left\{\left\{\sigma_{1}, \sigma_{2}\right\}\right\}(x)=\left\{\sigma_{1}(x), \sigma_{2}(x)\right\}+\mathrm{d} \sigma_{1}\left(\xi_{\sigma_{2}(x)}\right)-\mathrm{d} \sigma_{2}\left(\xi_{\sigma_{1}(x)}\right) .
$$

An anchor $\Psi: M \times \mathcal{G} \rightarrow T M$ is defined by $\Psi(x, X)=\xi_{X}(x)$. Then $\left(M \times \mathcal{G}, \operatorname{pr}_{1}, M, \Psi,\{\{\cdot, \cdot\}\}\right)$ is a Banach Lie algebroid.

Moreover, if we denote by $G_{x}$ the closed subgroup of isotropy of a point $x \in M$ and by $\mathcal{G}_{x} \subset \mathcal{G}$ its Lie subalgebra, we have $\operatorname{ker} \Psi_{x}=\mathcal{G}_{x}$. If $\mathcal{G}_{x}$ is complemented in $\mathcal{G}$ for any $x \in M$ and $\rho$ has closed range, then the weak distribution $\mathcal{D}=\Psi(M \times \mathcal{G})$ is integrable and the leaf through $x$ is its orbit $\psi(x, G)$ (cf. [26, Example 4.3,3]).

Note that in finite dimension it is classical that a Lie bracket $[\cdot, \cdot]_{E}$ on an anchored bundle $(E, \pi, M, \rho)$ respects the sheaf of sections of $\pi: E \rightarrow M$ or, for short, is localizable (see for instance [25]), if the following properties are satisfied:

(i) for any open set $U$ of $M$, there exists a unique bracket $[\cdot, \cdot]_{U}$ on the space of sections $\underline{E}_{\mid U}$ ) such that, for any $s_{1}$ and $s_{2}$ in $\left.\underline{E}_{\mid U}\right)$, we have:

$$
\left[s_{1 \mid U}, s_{1 \mid U}\right]_{U}=\left(\left[s_{1}, s_{2}\right]_{E}\right)_{\mid U}
$$

(ii) (compatibility with restriction) if $V \subset U$ are open sets, then, $[\cdot, \cdot]_{U}$ induces a unique Lie bracket $[\cdot, \cdot]_{U V}$ on $\left.\underline{E}_{\mid V}\right)$ which coincides with $[\cdot, \cdot]_{V}$ (induced by $[\cdot, \cdot]_{E}$ ).

By the same arguments as in finite dimension, when $M$ is smooth regular any Lie bracket $[\cdot, \cdot]_{E}$ on an anchored bundle $(E, \pi, M, \rho)$ is localizable (cf. $[26])$.

But, in general, for analog reasons as for Koszul connection, we can not prove that any Lie bracket is localizable. Unfortunately in the Banach framework, we have no example of Lie algebroid for which is not localizable. Therefore at least for finding conditions under which a Banach Lie algebroid is integrable this condition is necessary. This condition of localization implies also that a bracket depends on the one jets of sections. Therefore, in the sequel, we will assume that all almost Lie bracket $[\cdot, \cdot]_{E}$ are localizable.

Remark 6.7. - If there exists a Koszul connection $\nabla$ on $E$, then we get an almost Lie bracket $[\cdot, \cdot]_{\nabla}$ defined by

$$
\left[s_{1}, s_{2}\right]_{\nabla}=\nabla_{\rho s_{1}} s_{2}-\nabla_{\rho s_{2}} s_{1} .
$$


Note that since a $\nabla$ is localizable, so is $[\cdot, \cdot]_{\nabla}$

When $(E, \pi, M, \rho,[\cdot, \cdot])_{E}$ is an almost Banach Lie algebroid we can define the following operators:

(i) Lie derivative $L_{s}^{\rho}$ according to a section $s$ of $E$ : for a smooth function $f \in \Omega^{0}(M, E)=\mathcal{F}$,

$$
L_{s}^{\rho}(f)=L_{\rho \circ s}(f)=i_{\rho \circ s}(\mathrm{~d} f) ;
$$

for a $q$-form $\omega \in \Omega^{q}(M, E)$ (where $\left.q>0\right)$

$$
\begin{aligned}
\left(L_{s}^{\rho} \omega\right)\left(s_{1}, \ldots, s_{q}\right)=L_{s}^{\rho}( & \left.\omega\left(s_{1}, \ldots, s_{q}\right)\right) \\
& \quad-\sum_{i=1}^{q} \omega\left(s_{1}, \ldots, s_{i-1},\left[s, s_{i}\right]_{E}, s_{i+1}, \ldots, s_{q}\right) .
\end{aligned}
$$

(ii) $\Omega(M, E)$-value derivative according to a section $s$ of $E$ : for a smooth function $f \in \Omega^{0}(M, E)=\mathcal{F}$

$$
\mathrm{d}_{\rho} f=t_{\rho} \circ \mathrm{d} f
$$

for a $q$-form $\omega \in \Omega^{q}(M, E)$ (where $\left.q>0\right)$

$$
\begin{aligned}
\left(\mathrm{d}_{\rho} \omega\right)\left(s_{0}, \ldots, s_{q}\right) & =\sum_{i=0}^{q}(-1)^{i} L_{s_{i}}^{\rho}\left(\omega\left(s_{0}, \ldots, \widehat{s_{i}}, \ldots, s_{q}\right)\right) \\
& +\sum_{0 \leqslant i<j \leqslant q}^{q}(-1)^{i+j}\left(\omega\left(\left[s_{i}, s_{j}\right]_{E}, s_{0}, \ldots, \widehat{s_{i}}, \ldots, \widehat{s_{j}}, \ldots, s_{q}\right)\right) .
\end{aligned}
$$

In general, we have $\mathrm{d}_{\rho} \circ \mathrm{d}_{\rho} \neq 0$. However, $(E, M, \rho,[\cdot, \cdot])_{E}$ is a Banach Lie algebroid if and only if $\mathrm{d}_{\rho} \circ \mathrm{d}_{\rho}=0$.

Definition 6.8. - Let $\psi: E \rightarrow E^{\prime}$ be a linear bundle morphism over $f: M \rightarrow M^{\prime}$.

(1) A section $s^{\prime}$ of $E^{\prime} \rightarrow M^{\prime}$ and a section $s$ of $E \rightarrow M$ are $\psi$-related if $s^{\prime} \circ f=\psi \circ s$.

(2) $\psi$ is a morphism of almost Banach Lie algebroids from $(E, \pi, M, \rho$, $\left.[\cdot, \cdot]_{E}\right)$ to $\left(E^{\prime}, \pi^{\prime}, M^{\prime}, \rho^{\prime},[\cdot, \cdot]_{E^{\prime}}\right)$ if:

(a) $\rho^{\prime} \circ \psi=T f \circ \rho$;

(b) for any pair of $\psi$-related sections $s_{i}^{\prime}$ and $s_{i}(i=1,2)$, we have: $\psi\left(\left[s_{1}, s_{2}\right]\right)=\left[s_{1}^{\prime}, s_{2}^{\prime}\right]^{\prime} \circ f$, i.e. the Lie bracket $\left[s_{1}^{\prime}, s_{2}^{\prime}\right]^{\prime}$ and $\left[s_{1}, s_{2}\right]$ are $\psi$-related. 
In a dual way, a morphism $\psi: E \rightarrow E^{\prime}$ which satisfies property (a) is an almost Banach Lie algebroid morphism if the mapping $\psi^{*}: \Omega^{q}\left(M, E^{\prime}\right) \rightarrow$ $\Omega^{q}(M, E)$ defined by:

$$
\left(\psi^{*} \alpha^{\prime}\right)_{x}\left(s_{1}, \ldots, s_{q}\right)=\alpha_{f(x)}^{\prime}\left(\psi \circ s_{1}, \ldots, \psi \circ s_{q}\right)
$$

commutes with the differentials:

$$
\mathrm{d}_{\rho} \circ \psi^{*}=\psi^{*} \circ \mathrm{d}_{\rho^{\prime}} .
$$

Notice that an almost Banach Lie algebroid $\left(E, \pi, M, \rho,[\cdot, \cdot]_{E}\right)$ is a Banach algebroid if and only if the anchor $\rho$ is a morphism of Banach Lie algebroids from $\left(E, \pi, M, \rho,[\cdot, \cdot]_{E}\right)$ to the canonical Banach Lie algebroid $\left(T M, p_{M}, M, \operatorname{Id}_{T M},[\cdot, \cdot]\right)$.

\subsection{Direct limit of almost Banach Lie algebroids}

As in the Banach framework, if $\pi: E \rightarrow M$ is a convenient bundle over a n.n.H. convenient manifold $M$, then we can define the convenient algebroid or Lie algebroid structure ${ }^{(8)}\left(E, \pi, M, \rho,[\cdot, \cdot]_{E}\right)$ in an obvious way. Now coming back to the context of sequence of Banach anchored bundles, we have:

DeFinition 6.9.

(1) A sequence $\left(E_{n}, \pi_{n}, M_{n}, \rho_{n}\right)_{n \in \mathbb{N}^{*}}$ is called a strong ascending sequence of anchored Banach bundles if

(a) $\left(E_{n}, \pi_{n}, M_{n},\right)_{n \in \mathbb{N}^{*}}$ is a direct sequence of Banach bundles;

(b) For all $n \leqslant m$, we have

$$
\rho_{m} \circ \lambda_{n}^{m}=T \varepsilon_{n}^{m} \circ \rho_{n} .
$$

where $\lambda_{n}^{m}: E_{n} \rightarrow E_{m}$ and $\epsilon_{n}^{m}: M_{n} \rightarrow M_{m}$ are the bonding morphisms.

(2) A sequence $\left(E_{n}, \pi_{n}, M_{n}, \rho_{n},[\cdot, \cdot]_{n}\right)_{n \in \mathbb{N}^{*}}$ is called a strong ascending sequence of almost Banach Lie algebroids if $\left(E_{n}, \pi_{n}, M_{n}, \rho_{n}\right)_{n \in \mathbb{N}^{*}}$ is a strong ascending sequence of anchored Banach bundles with the additional property: $\lambda_{n}^{m}: E_{n} \rightarrow E_{m}$ is an almost Banach algebroid morphism between the almost Banach Lie algebroids $\left(E_{n}, \pi_{n}, M_{n}, \rho_{n},[\cdot, \cdot]_{n}\right)$ and $\left(E_{m}, \pi_{m}, M_{m}, \rho_{m},[\cdot, \cdot]_{m}\right)$.

(8) In this case $E$ has a structure of n.n.H. convenient manifold. 


\section{THEOREM 6.10.}

(1) If $\left.\left(E_{n}, \pi_{n}, M_{n}, \rho_{n}\right)\right)_{n \in \mathbb{N}^{*}}$ is a strong ascending sequence of anchored bundles, then $\left.\left(\lim _{\longrightarrow} E_{n}, \lim _{n} \pi_{n}, \lim _{\longrightarrow} M_{n}, \lim _{\longrightarrow} \rho_{n}\right)\right)$ is a convenient anchored bundle. Moreover, $\left(\stackrel{\lim }{E_{n}}, \lim _{\longrightarrow}, \lim _{n} M_{n}, \lim _{\longrightarrow} \rho_{n}, \varliminf_{\longrightarrow}[\cdot, \cdot]_{n}\right)$ is a convenient algebroid (resp. a convenient Lie algebroid) if each $\left(E_{n}, \pi_{n}, M_{n}, \rho_{n},[\cdot, \cdot]_{n}\right)$ is a Banach algebroid (resp. a Banach Lie algebroid) for $n \in \mathbb{N}^{*}$.

(2) If $\left(E_{n}, \pi_{n}, M_{n}, \rho_{n},[\cdot, \cdot]_{n}\right)_{n \in \mathbb{N}^{*}}$ is a strong ascending sequence of almost Banach Lie algebroids, then $\left(\lim _{\longrightarrow} E_{n}, \lim \pi_{n}, \underline{\lim } M_{n}, \underline{\lim } \rho_{n}\right.$, $\left.\underset{\longrightarrow}{\lim }[\cdot, \cdot]_{n}\right)$ is an almost convenient Lie algebroid. Moreover, $\left(\mathrm{lim}_{n} E_{n}, \lim _{\longrightarrow} \pi_{n}, \varliminf_{\longrightarrow} M_{n}, \lim _{\longrightarrow} \rho_{n}, \lim _{[}[\cdot, \cdot]_{n}\right)$ is a convenient algebroid (resp. a convenient Lie algebroid) if each $\left(E_{n}, \pi_{n}, M_{n}, \rho_{n},[\cdot, \cdot]_{n}\right)$ is a Banach algebroid (resp. a Banach Lie algebroid) for $n \in \mathbb{N}^{*}$.

\section{Proof.}

Step 1. - According to Proposition 4.18, $\left(\underline{\lim } E_{n}, \underline{\lim } \pi_{n}, \underline{\lim _{\longrightarrow}} M_{n}\right)$ can be endowed with a structure of convenient vector bundle whose base is modelled on the LB-space $\lim _{\longrightarrow} \mathbb{M}_{n}$ and whose structural group is the metrizable complete topological group $G(\mathbb{E})$.

Step 2. - Let $\left(s_{n}^{1}\right)_{n \in \mathbb{N}^{*}}$ and $\left(s_{n}^{2}\right)_{n \in \mathbb{N}^{*}}$ be sequences of sections of the linear bundles $\pi_{n}: E_{n} \rightarrow M_{n}$, i.e. fulfilling the conditions :

$$
\left\{\begin{array}{l}
\lambda_{n}^{m} \circ s_{n}^{1}=s_{m}^{1} \circ \varepsilon_{n}^{m} \\
\lambda_{n}^{m} \circ s_{n}^{2}=s_{m}^{2} \circ \varepsilon_{n}^{m} .
\end{array}\right.
$$

In order to define a structure of almost convenient Lie structure on the direct limit we have to prove the compatibility of the brackets

$$
\lambda_{n}^{m} \circ\left[s_{n}^{1}, s_{n}^{2}\right]_{E_{n}}=\left[s_{m}^{1}, s_{m}^{2}\right]_{E_{m}} \circ \varepsilon_{n}^{m}
$$

and the compatibility of the Leibniz properties:

$$
\lambda_{n}^{m} \circ\left[s_{n}^{1}, g_{n} \times s_{n}^{2}\right]_{E_{n}}=\left[s_{m}^{1}, g_{m} \times s_{m}^{2}\right]_{E_{m}} \circ \varepsilon_{n}^{m}
$$

Step 2a. - In order to prove (6.3) we use the morphisms $\lambda_{n}^{m}: E_{n} \rightarrow E_{m}$ of Lie algebroids over $\varepsilon_{n}^{m}: M_{n} \rightarrow M_{m}$ :

$$
\mathrm{d}_{\rho_{n}} \circ\left(\lambda_{n}^{m}\right)^{*}=\left(\lambda_{n}^{m}\right)^{*} \circ \mathrm{d}_{\rho_{m}}
$$

applied to $\alpha_{m} \in \Omega^{1}\left(M_{m}, E_{m}\right)$.

We then have $\left(\mathrm{d}_{\rho_{n}} \circ\left(\lambda_{n}^{m}\right)^{*}\left(\alpha_{m}\right)\right)\left(s_{n}^{1}, s_{n}^{2}\right)=\left(\left(\lambda_{n}^{m}\right)^{*} \circ \mathrm{d}_{\rho_{m}}\left(\alpha_{m}\right)\right)\left(s_{n}^{1}, s_{n}^{2}\right)$, 
For the LHS, we have:

$$
\begin{aligned}
\left(\mathrm{d}_{\rho_{n}} \circ\right. & \left.\left(\lambda_{n}^{m}\right)^{*}\left(\alpha_{m}\right)\right)\left(s_{n}^{1}, s_{n}^{2}\right) \\
& =L_{\rho_{n} \circ s_{n}^{1}}\left(\left(\left(\lambda_{n}^{m}\right)^{*}\left(\alpha_{m}\right)\right)\left(s_{n}^{2}\right)\right)-L_{\rho_{n} \circ s_{n}^{2}}\left(\left(\left(\lambda_{n}^{m}\right)^{*}\left(\alpha_{m}\right)\right)\left(s_{n}^{1}\right)\right) \\
& \quad-\left(\left(\lambda_{n}^{m}\right)^{*}\left(\alpha_{m}\right)\right)\left[s_{n}^{1}, s_{n}^{2}\right]_{E_{n}} \\
& =X_{m}^{1}\left(\alpha_{m}\left(\lambda_{n}^{m} \circ s_{n}^{2}\right)\right)-X_{m}^{2}\left(\alpha_{m}\left(\lambda_{n}^{m} \circ s_{m}^{1}\right)\right)-\alpha_{m}\left(\lambda_{n}^{m} \circ\left[s_{n}^{1}, s_{n}^{2}\right]_{E_{n}}\right)
\end{aligned}
$$

where $X_{m}^{a}=\rho_{m} \circ s_{m}^{a}$ with $a=1,2$ fulfill the relation $X_{m}^{a}\left(f_{m}\right)=X_{n}^{a}\left(f_{n}\right)$ for $f_{m}=\alpha_{m} \circ s_{m}$.

For the RHS, we get:

$$
\begin{aligned}
& \left(\left(\lambda_{n}^{m}\right)^{*}\left(\mathrm{~d}_{\rho_{m}}\left(\alpha_{m}\right)\right)\right)\left(s_{n}^{1}, s_{n}^{2}\right) \\
& =\mathrm{d}_{\rho_{m}}\left(\alpha_{m}\right)\left(\lambda_{n}^{m} \circ s_{n}^{1}, \lambda_{n}^{m} \circ s_{n}^{2}\right) . \\
& =L_{\rho_{m} \circ \lambda_{n}^{m} \circ s_{n}^{1}}\left(\alpha_{m}\left(\lambda_{n}^{m} \circ s_{n}^{2}\right)\right)-L_{\rho_{m} \circ \lambda_{n}^{m} \circ s_{n}^{2}}\left(\alpha_{m}\left(\lambda_{n}^{m} \circ s_{n}^{1}\right)\right) \\
& \quad-\alpha_{m}\left[\lambda_{n}^{m} \circ s_{n}^{1}, \lambda_{n}^{m} \circ s_{n}^{2}\right]_{E_{m}} \\
& =L_{\rho_{m} \circ s_{m}^{1}}\left(\alpha_{m}\left(\lambda_{n}^{m} \circ s_{n}^{2}\right)\right)-L_{\rho_{m} \circ s_{m}^{2}}\left(\alpha_{m}\left(\lambda_{n}^{m} \circ s_{n}^{1}\right)\right)-\alpha_{m}\left[\lambda_{n}^{m} \circ s_{n}^{1}, \lambda_{n}^{m} \circ s_{n}^{2}\right]_{E_{m}} \\
& =X_{m}^{1}\left(\alpha_{m}\left(\lambda_{n}^{m} \circ s_{n}^{2}\right)\right)-X_{m}^{2}\left(\alpha_{m}\left(\lambda_{n}^{m} \circ s_{m}^{1}\right)\right)-\alpha_{m}\left[\lambda_{n}^{m} \circ s_{n}^{1}, \lambda_{n}^{m} \circ s_{n}^{2}\right]_{E_{m}} .
\end{aligned}
$$

Finally, we have for all $\alpha_{m} \in \Omega^{1}\left(M_{m}, E_{m}\right), \alpha_{m}\left(\lambda_{n}^{m}\left(\left[s_{n}^{1}, s_{n}^{2}\right]_{E_{n}}\right)\right)=$ $\alpha_{m}\left[\lambda_{n}^{m} \circ s_{n}^{1}, \lambda_{n}^{m} \circ s_{n}^{2}\right]_{E_{m}}$ and we obtain: $\lambda_{n}^{m} \circ\left[s_{n}^{1}, s_{n}^{2}\right]_{E_{n}}=\left[\lambda_{n}^{m} \circ s_{n}^{1}, \lambda_{n}^{m} \circ s_{n}^{2}\right]_{E_{m}}$. Using $\lambda_{n}^{m} \circ s_{n}^{a}=s_{m}^{a} \circ \varepsilon_{n}^{m}$, we have: $\lambda_{n}^{m} \circ\left[s_{n}^{1}, s_{n}^{2}\right]_{E_{n}}=\left[s_{m}^{1}, s_{m}^{2}\right]_{E_{m}} \circ \varepsilon_{n}^{m}$.

Step 2b. - To prove (6.4) we are going to establish that

$$
\begin{aligned}
\lambda_{n}^{m} \circ\left(g_{n} \times\left[s_{n}^{1}, s_{n}^{2}\right]_{E_{n}}+\right. & \left.\left(\rho_{n}\left(s_{n}^{1}\right)\right)\left(g_{n}\right) \times s_{n}^{2}\right) \\
& =\left(g_{m} \times\left[s_{m}^{1}, s_{m}^{2}\right]_{E_{m}}+\left(\rho_{m}\left(s_{m}^{1}\right)\right)\left(g_{m}\right) \times s_{m}^{2}\right) \circ \varepsilon_{n}^{m}
\end{aligned}
$$

We can write:

$$
\begin{aligned}
& \lambda_{n}^{m} \circ\left(g_{n} \times\left[s_{n}^{1}, s_{n}^{2}\right]_{E_{n}}+\left(\rho_{n}\left(s_{n}^{1}\right)\right)\left(g_{n}\right) \times s_{n}^{2}\right) \\
& =\lambda_{n}^{m} \circ\left(g_{n} \times\left[s_{n}^{1}, s_{n}^{2}\right]_{E_{n}}\right)+\lambda_{n}^{m} \circ\left(\left(\rho_{n}\left(s_{n}^{1}\right)\right)\left(g_{n}\right) \times s_{n}^{2}\right) \\
& =g_{n} \times\left(\lambda_{n}^{m} \circ\left[s_{n}^{1}, s_{n}^{2}\right]_{E_{n}}\right)+\lambda_{n}^{m}\left(X_{n}^{1}\left(g_{n}\right)\right) \times \lambda_{n}^{m} \circ s_{n}^{2} \quad\left(\lambda_{n}^{m} \text { is a morphism }\right) \\
& =g_{n} \times\left(\left[s_{m}^{1}, s_{m}^{2}\right]_{E_{m}} \circ \varepsilon_{n}^{m}\right)+X_{m}^{1}\left(g_{m}\right) \circ \varepsilon_{n}^{m} \times s_{m}^{2} \circ \varepsilon_{n}^{m} \quad \text { cf. }(6.3) \\
& =\left(g_{m} \circ \varepsilon_{n}^{m}\right) \times\left(\left[s_{m}^{1}, s_{m}^{2}\right]_{E_{m}} \circ \varepsilon_{n}^{m}\right)+\left(X_{m}^{1}\left(g_{m}\right) \times s_{m}^{2}\right) \circ \varepsilon_{n}^{m} \\
& =\left(g_{m} \times\left[s_{m}^{1}, s_{m}^{2}\right]_{E_{m}}\right) \circ \varepsilon_{n}^{m}+\left(\rho_{m}\left(s_{m}^{1}\right)\left(g_{m}\right) \times s_{m}^{2}\right) \circ \varepsilon_{n}^{m} \\
& =\left(g_{m} \times\left[s_{m}^{1}, s_{m}^{2}\right]_{E_{m}}+\left(\rho_{m}\left(s_{m}^{1}\right)\right)\left(g_{m}\right) \times s_{m}^{2}\right) \circ \varepsilon_{n}^{m} .
\end{aligned}
$$

Step 3. - Now, from the previous construction of $\lim [\cdot, \cdot]_{n}$, it is clear that if $\rho_{n}$ is a morphism of almost algebroids from $\left(E_{n}, \pi_{n}, M_{n}, \rho_{n},[\cdot, \cdot]_{n}\right)$ to 
the canonical Banach Lie algebroid $\left(T M_{n}, \pi_{n}, M_{n}, \operatorname{Id}_{T M_{n}},[\cdot, \cdot]\right)$, then $\lim _{\longrightarrow} \rho_{n}$ satisfies

$$
\underset{\lim }{\longrightarrow} \rho_{n}\left(\lim _{\longrightarrow}[\cdot, \cdot]_{n}\right)=\left[\lim _{\longrightarrow} \rho_{n}(\cdot), \lim _{\longrightarrow} \rho_{n}(\cdot)\right] .
$$

Moreover, it is also easy to show that if each bracket $[\cdot, \cdot]_{n}$ satisfies the Jacobi identity, then $\lim [\cdot, \cdot]_{n}$ satisfies also a Jacobi identity. These last proofs are left to the reader.

Corollary 6.11. - Let $\left(D_{n}\right)_{n \in \mathbb{N}^{*}}$ be a strong ascending sequence of Banach connections on a strong ascending sequence $\left(E_{n}, \pi_{n}, M_{n}\right)_{n \in \mathbb{N}^{*}}$ of $B a$ nach bundles. Then there exists an almost convenient Lie algebroid structure on the bundle $\left(\lim _{\longrightarrow} E_{n}, \lim _{n} \pi_{n}, \lim _{\longrightarrow} M_{n}\right)$.

Proof. - On each anchored bundle $\left(E_{n}, \pi_{n}, M_{n}, \rho_{n}\right)$, we denote by $\nabla_{n}$ the $E_{n}$-Koszul connection associated to $D_{n}$. Therefore $\left[s_{n}^{1}, s_{n}^{2}\right]_{n}=$ $\nabla_{\rho_{n}\left(s_{n}^{1}\right)}^{n} s_{n}^{2}-\nabla_{\rho_{n}\left(s_{n}^{2}\right)}^{n} s_{n}^{1}$ defines an almost Lie bracket on $\left(E_{n}, \pi_{n}, M_{n}, \rho_{n}\right)$. Since $\left(D_{n}\right)_{n \in \mathbb{N}^{*}}$ is a direct sequence of Banach connections, it follows that the sequence $\left([\cdot, \cdot]_{n}\right)_{n \in \mathbb{N}^{*}}$ of almost brackets satisfies a property of Definition 6.9. Therefore, from Theorem $6.10, \underline{\lim }[\cdot, \cdot]_{n}$ is an almost Lie bracket on the convenient anchored bundle $\left(\stackrel{\lim }{\longrightarrow} E_{n}, \underline{\lim } \pi_{n}, \underline{\longrightarrow} M_{n}, \lim _{\longrightarrow} \rho_{n}\right)$.

\section{Integrability of distributions which are direct limit of local Koszul Banach bundles}

\subsection{Integrability of the range of an anchor}

We first recall the classical definitions of distribution, integrability and involutivity.

DeFinition 7.1. - Let $M$ be a Banach manifold.

(1) A distribution $\Delta$ on $M$ is an assignment $\Delta: x \mapsto \Delta_{x} \subset T_{x} M$ on $M$ where $\Delta_{x}$ is a subspace of $T_{x} M$.

(2) A vector field $X$ on $M$, defined on an open set $\operatorname{Dom}(X)$, is called tangent to a distribution $\Delta$ if $X(x)$ belongs to $\Delta_{x}$ for all $x \in$ $\operatorname{Dom}(X)$.

(3) A distribution $\Delta$ on $M$ is called integrable if, for all $x_{0} \in M$, there exists a weak submanifold $(N, \phi)$ of $M$ such that $\phi\left(y_{0}\right)=x_{0}$ for some $y_{0} \in N$ and $T \phi\left(T_{y} N\right)=\Delta_{\phi(y)}$ for all $y \in N$. In this case $(N, \phi)$ is called an integral manifold of $\Delta$ through $x$.

(4) A distribution $\Delta$ is called involutive if for any vector fields $X$ and $Y$ on $M$ tangent to $\Delta$ the Lie bracket $[X, Y]$ defined on $\operatorname{Dom}(X) \cap$ $\operatorname{Dom}(Y)$ is tangent to $\Delta$. 
Classically, in Banach context, when $\Delta$ is a complemented subbundle of $T M$, according to the Frobenius Theorem, involutivity implies integrability.

In finite dimension, the famous results of H. Sussman and P. Stefan give necessary and sufficient conditions for the integrability of smooth distributions.

A generalization of these results in the context of Banach manifolds can be found in [5] and [26].

We are now in a position to prove the following theorem which will be useful for the proof of the main theorem on the integrability of a distribution on a direct limit of Banach manifolds endowed with Koszul connections.

Theorem 7.2. - Let $\left(E, \pi, M, \rho,[\cdot, \cdot]_{E}\right)$ be a Banach algebroid (cf. Subsection 6.1). Assume that for each $x \in M$, the kernel of $\rho_{x}$ is complemented in each fiber $E_{x}$ and $\mathcal{D}_{x}=\rho\left(E_{x}\right)$ is closed in $T_{x} M$. Then $\mathcal{D}$ is an integrable weak distribution of $M$.

Assume that there exists a linear connection on E. Then there exists a non linear connection on the tangent bundle of each leaf of the distribution $\mathcal{D}$.

Proof. - The first part of this theorem is an easy adaptation of the proof of Theorem 5 in [26].

We consider a leaf $L$ of $\mathcal{D}$. If $\iota: L \rightarrow M$ is the natural inclusion, it is a smooth immersion of $L$ in $M$. Let $x$ be any point of $L$ and denote $K_{x}$ the kernel of $\rho_{x}: \pi^{-1}(x)=E_{x} \rightarrow T_{x} M$. According to the assumption on $E$, we have a decomposition $E_{x}=K_{x} \oplus F_{x}$. From the proof of Theorem 2 of [26], $L$ is a Banach manifold modelled on $F:=F_{x}$. Consider the pull back $E_{L}$ of $E$ over $L$ via $\iota: L \rightarrow M$. We have a bundle morphism $\hat{\iota}$ from $E_{L}$ in $E$ over $\iota$ which is an isomorphism on each fiber. Therefore, the kernel of $\hat{\rho}=\rho \circ \iota$ is a Banach subbundle $K_{L}$ of $E_{L}$ and we have a subbundle $F_{L}$ of $E_{L}$ such that $E_{L}=K_{L} \oplus F_{L}$. In particular, we have an isomorphism $\rho_{L}$ from $F_{L}$ to TL. It follows that the tangent map $T \rho_{L}: T F_{L} \rightarrow T(T L)$ is also an isomorphism. On the other hand, according to the decomposition $E_{L}=K_{L} \oplus F_{L}$, we have also a decomposition $T E_{L}=T K_{L} \oplus T F_{L}$.

Now, assume that there exists a non linear connection on $E$ and let $D$ : $T E \rightarrow E$ be the associated map connection. The map $\widehat{D}_{L}=D \circ \hat{\imath} \circ\left(T \rho_{L}\right)^{-1}$ is smooth and maps the fiber of $T_{(x, u)}(T L)$ over $(x, u) \in T L$ into the fiber of $\left(E_{L}\right)_{x}$ over $x \in L$. As $\hat{\iota}$ is an isomorphism from $\left(E_{L}\right)_{x}$ to the fiber $E_{x}$ over $\iota(x)$, it follows that $\widehat{D}_{L}$ is a linear continuous map between these fibers. In particular, we can consider $\widehat{D}_{L}$ as a map from $T(T L)$ into $E_{L}$. Now if $\Pi_{L}$ is the projection of $E_{L}$ on $F_{L}$ parallel to $K_{L}$, the map $D_{L}=\rho_{L} \circ \Pi_{L} \circ \widehat{D}_{L}$ defines a Koszul connection on TL. 


\subsection{Criterion of integrability for local direct limits of local Koszul Banach bundles}

Let $M$ be a n.n.H. convenient manifold and denote by $T M$ its dynamical tangent bundle. In the same way, a distribution $\Delta$ on $M$ is again an assignment $\Delta: x \mapsto \Delta_{x} \subset T_{x} M$ on $M$ where $\Delta_{x}$ is a subspace of $T_{x} M$. The notion of integrability and involutivity of a distribution recalled in Subsection 7.1 can be clearly adapted to the convenient context.

We will now give a criterion of integrability for direct limit of local Koszul Banach bundles. More precisely we have:

Definition 7.3. - A distribution $\Delta$ on a n.n.H. convenient manifold $M$ is called a local direct limit of local Koszul Banach bundles if the following property is satisfied:

(*) for any $x \in M$, there exists an open neighbourhood $U$ of $x$ and a strong ascending sequence of anchored Banach bundles $\left(E_{n}, \pi_{n}, U_{n}, \rho_{n}\right)_{n \in \mathbb{N}^{*}}$ endowed with a Koszul connection $\nabla^{n}$ such that $U=\lim _{n} U_{n}, \lim _{n} \rho_{n}\left(E_{n}\right)=\Delta_{\mid U}$ and such that $E_{n}$ is a complemented subbundle of $\vec{E}_{n+1}$.

Remark 7.4. - In the context of paracompact finite dimensional manifolds or Hilbert manifolds, the condition of the existence of a Koszul connection $\nabla^{n}$ and $E_{n}$ complemented in $E_{n+1}$ are automatically satisfied.

We then have the following criterion of integrability:

TheOREm 7.5. - Let $\Delta$ be a local direct limit of local Koszul Banach bundles. Assume that in the property $(*)$ there exists an almost Lie bracket $[\cdot, \cdot]_{n}$ on $\left(E_{n}, \pi_{n}, U_{n}, \rho_{n}\right)$ such that $\left(E_{n}, \pi_{n}, U_{n}, \rho_{n},[\cdot, \cdot]_{n}\right)$ is a Banach algebroid, and over each point $y_{n} \in U_{n}$ the kernel of $\rho_{n}$ is complemented in the fiber $\pi_{n}^{-1}\left(y_{n}\right)$ and the range of $\rho_{n}$ is closed.

Then the distribution $\Delta$ is integrable and the maximal integral manifold $N$ through $x=\lim _{\longrightarrow} x_{n}$ is a weak n.n.H. convenient submanifold of $M$ which is a direct limit of the set of maximal leaves $N_{n}$ of $\rho_{n}\left(E_{n}\right)$ through $x_{n}$ in $M_{n}$. Moreover, each maximal leaf has the limit chart property at any point and if $M$ is Hausdorff so is each leaf.

Proof. - At first, for each $n \in \mathbb{N}^{*}$, we can apply the first part of Theorem 7.2. Therefore, with the notation of property $(*)$, if we fix some $x=$ $\stackrel{\lim }{\longrightarrow} x_{n}$, there exists a maximal integral manifold $N_{n}$ of $\rho_{n}\left(E_{n}\right)$ through $x_{n}$ $\overrightarrow{\text { in }} U_{n}$. Recall that we have $U_{n} \subset U_{n+1}$ and $E_{n} \subset E_{n+1}$ over $U_{n}$. Therefore, according to Property (2) of Definition 6.9, for any $y \in N_{n}$, we have 
$T_{y} N_{n} \subset T_{y} N_{n+1}$ on $N_{n} \cap N_{n+1}$. Since $N_{n+1}$ is a maximal integral manifold of $\rho_{n+1}\left(E_{n+1}\right)$ in $U_{n+1}$ and $U_{n} \subset U_{n+1}$, if $y$ belongs to $N_{n}$, we have a smooth curve in $N_{n}$ which joins $x_{n}$ to $y$ and since $E_{n} \subset E_{n+1}$ over $U_{n}$ this curve must be contained in $N_{n+1}$ and so $N_{n}$ must be contained in $N_{n+1}$. Now, on the one hand, over each point of $U_{n}$ the kernel of $\rho_{n}$ is complemented in each fiber and, on the other hand, over $N_{n}$ the kernel of $\rho_{n}$ is a subbundle of $E_{n \mid N_{n}}$. The same property is true for $E_{n+1 \mid N_{n+1}}$. But over $N_{n} \subset N_{n+1}$, we have

$$
\rho_{n}\left(E_{n \mid N_{n}}\right)=T N_{n} \subset\left(T N_{n+1}\right)_{\mid N_{n}}=\rho_{n+1}\left(E_{n+1 \mid N_{n}}\right) .
$$

Therefore $\left(\operatorname{ker} \rho_{n+1}\right)_{\mid N_{n}} \subset\left(\operatorname{ker} \rho_{n}\right)_{\mid N_{n}}$. But, from our assumption, we have the following Whitney decomposition:

$$
E_{n+1 \mid N_{n}}=F_{n+1} \oplus\left(\operatorname{ker} \rho_{n+1}\right)_{\mid N_{n}} \text { and } \quad E_{n \mid N_{n}}=F_{n} \oplus\left(\operatorname{ker} \rho_{n}\right)_{\mid N_{n}} .
$$

Therefore

$$
\left(\operatorname{ker} \rho_{n}\right)_{\mid N_{n}}=\left(\operatorname{ker} \rho_{n+1}\right)_{\mid N_{n}} \oplus F_{n+1} \cap\left(\operatorname{ker} \rho_{n}\right)_{\mid N_{n}}
$$

Finally we obtain:

$$
\left(T N_{n+1}\right)_{\mid N_{n}}=T N_{n} \oplus \rho_{n+1}\left(F_{n+1} \cap\left(\operatorname{ker} \rho_{n}\right)_{\mid N_{n}}\right) .
$$

Now, from property $(*)$ and the second part of Theorem 7.2 , we have a linear connection on $T N_{n}$. Thus the ascending sequence $\left(N_{n}\right)$ satisfies the assumption of Corollary 4.9, $N=\underline{\lim } N_{n}$ has a structure of convenient manifold modelled on an LB-space. Moreover, by construction, we have $T N=\Delta_{\mid N}$. This means that $\Delta$ is an integral manifold of $\Delta$ through $x$. Moreover, $N$ satisfies the direct limit chart property.

Take any maximal integral manifold $L$ of $\Delta$ and choose some $x=\lim _{\longrightarrow} x_{n}$ in $L$. From our previous construction we have a sequence of Banach integral manifolds $\left(N_{n}\right)$ such that $N=\lim N_{n}$ is an integral manifold of $\Delta$ through $x$. Therefore $N$ is open in $L$. Since $N$ has the direct limit chart property, the same is true of $L$.

Now as the intersection of an open set in $M$ with any leaf $L$ is an open set of $L$, thus, if $M$ is an Hausdorff topological space, $L$ inherits of this property.

From this result we easily obtain:

Corollary 7.6. - Let $\left(E_{n}, \pi_{n}, M_{n}, \rho_{n},[\cdot, \cdot]_{n}\right)_{n \in \mathbb{N}^{*}}$ be a strong ascending sequence of Banach algebroids provided with a Koszul connection on each $E_{n}$ such that over each point $x_{n} \in M_{n}$ the kernel of $\rho_{n}$ is complemented in the fiber $\pi_{n}^{-1}\left(x_{n}\right)$ and the range of $\rho_{n}$ is closed. Then $\Delta=\lim _{\longrightarrow} \rho_{n}\left(E_{n}\right)$ is an integrable distribution on $M=\underline{\lim } M_{n}$. Moreover, for any $\vec{x}=\underline{\lim _{\longrightarrow}} x_{n}$, the 
maximal leaf through $x$ is a weak n.n.H. convenient submanifold of $M$ and there exists a leaf $N_{n}$ of $\rho_{n}\left(E_{n}\right)$ in $M_{n}$ through $x_{n}$ such that the sequence $\left(N_{n}\right)_{n \in \mathbb{N}^{*}}$ is an ascending sequence of Banach manifolds whose direct limit $N=\lim _{n} N_{n}$ is an integral manifold of $\Delta$ through $x$ such that $N$ has the direct limit chart property at $x$. Moreover, if $M$ is Hausdorff so is each leaf.

Now according to Remark 7.4 we also easily obtain:

Corollary 7.7. - Let $\Delta$ be a distribution on a direct limit $M=$

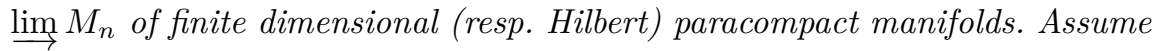
that, for any $x=\lim _{\longrightarrow} x_{n}$, there exists a sequence of finite rank (resp. Hilbert) algebroids $\left(E_{n}, \pi_{n}, U_{n}, \rho_{n}\right)_{n \in \mathbb{N}^{*}}$ such that $U=\lim _{\longrightarrow} U_{n}, \underline{\lim } \rho_{n}\left(E_{n}\right)=\Delta_{\mid U}$. Then $\Delta$ is integrable and the maximal integral manifold $N$ through $x=$ $\lim _{\longrightarrow} x_{n}$ is a weak convenient submanifold of $M$ which is the direct limit of the set of maximal leaves $N_{n}$ of $\rho_{n}\left(E_{N}\right)$ through $x_{n}$ in $M_{n}$. Moreover, each maximal leaf has the limit chart property at any point and is a Hausdorff convenient manifold.

\subsection{Application}

Consider a direct sequence of Banach Lie groups $G_{1} \subset G_{2} \subset \cdots \subset G_{n} \subset$ ... such that the Lie algebra $\mathcal{G}_{n}$ is complemented in the Lie algebra $\mathcal{G}_{n+1}$ for all $n \in \mathbb{N}^{*}$.

Note that this situation always occurs if for all $n \in \mathbb{N}^{*}$, each Lie group $G_{n}$ is finite dimensional or is a Hilbert Lie group. This assumption is also valid for the sequence $G_{n}=G L\left(E_{n}\right)$ where $E_{n}$ is a direct sequence of Banach spaces such that each $E_{n}$ is closed and complemented in $E_{n+1}$.

According to Example 6.6, assume that for each $n \in \mathbb{N}^{*}$, we have a smooth right action $\psi_{n}: M_{n} \times G_{n} \rightarrow M_{n}$ of $G_{n}$ over a Banach manifold $M_{n}$ where $M_{1} \subset M_{2} \subset \cdots \subset M_{n} \subset \ldots$ is an ascending sequence such that $M_{n}$ is a Banach submanifold of $M_{n+1}$. We get a strong ascending sequence Lie Banach algebroids $\left(M_{n} \times \mathcal{G}_{n}, \pi_{n}, M_{n}, \Psi_{n},[\cdot, \cdot]_{\mathcal{G}_{n}}\right)$. Since each Banach bundle $M_{n} \times \mathcal{G}_{n}$ is trivial, we obtain a sequence of Banach Lie algebroids with anchors

$$
\begin{aligned}
\Psi_{n}: M_{n} \times \mathcal{G}_{n} & \longrightarrow T M_{n} \\
\left(x_{n}, X_{n}\right) & \longmapsto T_{\left(x_{n}, e_{n}\right)} \psi_{n}\left(0, X_{n}\right)
\end{aligned}
$$

Because these bundles are trivial, we get a sequence of compatible trivial Koszul connections $\nabla^{n}$ on $M_{n} \times \mathcal{G}_{n}$. Now, from the Corollary 7.6, we obtain: 
THEOREM 7.8. - In the previous context, we obtain a smooth right action $\psi=\underline{\lim } \psi_{n}$ of $G=\underline{\lim } G_{n}$ on the convenient manifold $M=\underline{\lim } M_{n}$. Moreover, if the kernel of $\Psi_{n}$ is complemented in each fiber $\pi_{n}^{-1}\left(x_{n}\right)$ and the range of $\Psi_{n}$ is closed, then the orbit $\psi(x, G)$ of this action through $x=\underline{\lim } x_{n}$ is a weak n.n.H. convenient submanifold of $M$ which is the direct limit of the set of $G_{n}$-orbits $\left\{\psi_{n}\left(x_{n}, G_{n}\right)\right\}_{n \in \mathbb{N}^{*}}$. If $M$ is Hausdorff, so is each orbit.

Acknowledgments. The authors would like to thank the anonymous reviewer for providing valuable comments and suggestions

\section{Bibliography}

[1] R. Abraham, J. E. Marsden \& T. Ratiu, Manifolds, tensor analysis, and applications, 2nd ed., Applied Mathematical Sciences, vol. 75, Springer, 1988.

[2] M. Anastasiei, "Banach Lie algebroids", An. Ştiinţ. Univ. Al. I. Cuza Iaşi. Mat. (N.S.) 57 (2011), no. 2, p. 409-416.

[3] J. Boman, "Differentiability of a function and of its compositions with functions of one variable", Math. Scand. 20 (1967), p. 249-268.

[4] N. Bourbaki, Éléments de mathématique. Algèbre. Chapitres 1 à 3, 2nd ed., Springer, 2006.

[5] D. Chillingworth \& P. Stefan, "Integrability of singular distributions on Banach manifolds", Math. Proc. Camb. Philos. Soc. 79 (1976), no. 1, p. 117-128.

[6] J. Cortés, M. De León, J. C. Marrero \& E. Martínez, "Non holonomic Lagrangian systems on Lie algebroids", Discrete Contin. Dyn. Syst. 24 (2009), no. 2, p. 213-271.

[7] A. B. Cruzeiro \& S. Fang, "Weak Levi-Civita connection for the damped metric on the Riemannian path space and vanishing of Ricci tensor in adapted differential geometry", J. Funct. Anal. 185 (2001), no. 2, p. 681-698.

[8] R. DAhmen, "Direct limit constructions in infinite dimensional Lie theory", PhD Thesis, Universität Paderborn (Germany), 2011, http://nbn-resolving.de/urn:nbn: de: hbz: 466:2-239.

[9] C. T. J. Dodson \& G. N. Galanis, "Second order tangent bundles of infinite dimensional manifolds", J. Geom. Phys. 52 (2004), no. 2, p. 127-136.

[10] A. Frölicher \& A. Kriegl, Linear spaces and differentiation theory, Pure and Applied Mathematics, John Wiley \& Sons, 1988.

[11] H. Glöckner, "Direct limit Lie groups and manifolds", J. Math. Kyoto Univ. 43 (2003), no. 1, p. 2-26.

[12] , "Fundamentals of direct limit Lie theory", Compos. Math. 141 (2005), no. 6, p. $1551-1577$.

[13] — , "Direct limits of infinite-dimensional Lie groups compared to direct limits in related categories", https://arxiv.org/abs/math/0606078, 2006.

[14] - "Direct limits of infinite-dimensional Lie groups compared to direct limits in related categories", J. Funct. Anal. 245 (2007), no. 1, p. 19-61.

[15] J. Grabowski \& M. Jóźwikowski, "Pontryagin maximum principle on almost Lie algebroids", SIAM J. Control Optimization 49 (2011), no. 3, p. 1306-1357.

[16] D. W. Hajek \& G. E. Strecker, Direct limits of Hausdorff spaces, Academia, Prague, 1972, 165-169 pages. 
[17] V. L. HANSEn, "Some theorems on direct limits of expanding sequences of manifolds", Math. Scand. 29 (1971), p. 5-36.

[18] H. Herrlich, "Separation axioms and direct limits", Can. Math. Bull. 12 (1969), p. 337-338.

[19] T. Hirai, H. Shimomura, N. Tatsuuma \& E. Hirai, "Inductive limits of topologies, their direct products, and problems related to algebraic structures", J. Math. Kyoto Univ. 41 (2001), no. 3, p. 475-505.

[20] M. V. KARASËv, "Analogues of the objects of Lie group theory for nonlinear Poisson brackets", Math. USSR, Izv. 28 (1987), p. 497-527.

[21] A. Kriegl \& P. W. Michor, The convenient setting of global analysis, Mathematical Surveys and Monographs, vol. 53, American Mathematical Society, 1997.

[22] S. Lang, Differential and Riemannian manifolds, 3rd ed., Graduate Texts in Mathematics, vol. 160, Springer, 1995.

[23] M. de León, J. C. Marrero \& D. Martín de Diego, "Linear almost Poisson structures and Hamilton-Jacobi equation. Applications to nonholonomic mechanics", J. Geom. Mech. 2 (2010), no. 2, p. 159-198.

[24] G. Loaiza \& H. R. Quiceno, "A q-exponential statistical Banach manifold", J. Math. Anal. Appl. 398 (2013), no. 2, p. 466-476.

[25] C.-M. Marle, "Differential calculus on a Lie algebroid and Poisson manifolds", in The J. A. Pereira da Silva birthday schrift, Textos de Matemática. Série B., vol. 32, Universidade de Coimbra, 2002, p. 83-149.

[26] F. Pelletier, "Integrability of weak distributions on Banach manifolds", Indag. Math., New Ser. 23 (2012), no. 3, p. 214-242.

[27] P. PÉrez Carreras \& J. Bonet, Barrelled locally convex spaces, North-Holland Mathematics Studies, vol. 131, North-Holland, 1987.

[28] P. Popescu \& M. Popescu, "Anchored vector bundles and Lie algebroids", in Lie algebroids and related topics in differential geometry (Warsaw, 2000), Banach Center Publications, vol. 54, Polish Academy of Sciences, 2001, p. 51-69.

[29] J. Pradines, "Théorie de Lie pour les groupoïdes différentiables. Relations entre propriétés locales et globales", C. R. Math. Acad. Sci. Paris 263 (1966), p. 907-910.

[30] P. Stefan, "Integrability of systems of vector fields", J. Lond. Math. Soc. 21 (1980), no. 3, p. 544-556.

[31] H. J. Sussmann, "Orbits of families of vector fields and integrability of distributions", Trans. Am. Math. Soc. 180 (1973), p. 171-188.

[32] J. Vilms, "Connections on tangent bundles", J. Differ. Geom. 1 (1967), p. 235-243.

[33] A. Weinstein, "Symplectic groupoids and Poisson manifolds", Bull. Am. Math. Soc. 16 (1987), no. 1, p. 101-104. 